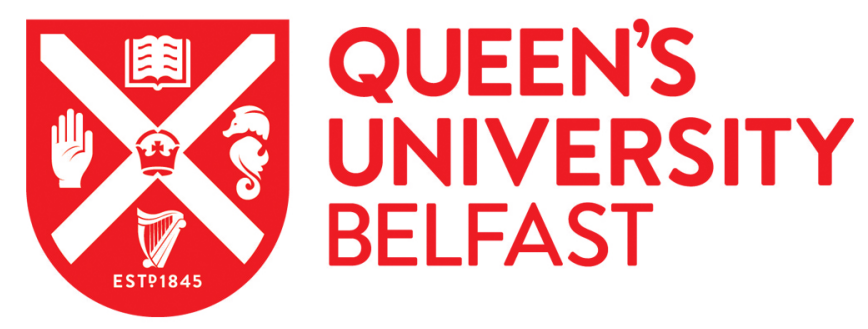

\title{
Real-Time Monitoring of Biopharmaceutical Crystallization: Chord Length Distribution to Crystal Size Distribution for Lysozyme, rHu Insulin, and Vitamin B12
}

Pandit, A., Katkar, V., Ranade, V., \& Bhambure, R. (2019). Real-Time Monitoring of Biopharmaceutical Crystallization: Chord Length Distribution to Crystal Size Distribution for Lysozyme, rHu Insulin, and Vitamin B12. Industrial and Engineering Chemistry Research, 58(18), 7607-7619.

https://doi.org/10.1021/acs.iecr.8b04613, https://doi.org/10.1021/acs.iecr.8b04613

Published in:

Industrial and Engineering Chemistry Research

Document Version:

Peer reviewed version

Queen's University Belfast - Research Portal:

Link to publication record in Queen's University Belfast Research Portal

\footnotetext{
Publisher rights

(c) 2018 American Chemical Society. This work is made available online in accordance with the publisher's policies. Please refer to any applicable terms of use of the publisher.
}

\section{General rights}

Copyright for the publications made accessible via the Queen's University Belfast Research Portal is retained by the author(s) and / or other copyright owners and it is a condition of accessing these publications that users recognise and abide by the legal requirements associated with these rights.

Take down policy

The Research Portal is Queen's institutional repository that provides access to Queen's research output. Every effort has been made to ensure that content in the Research Portal does not infringe any person's rights, or applicable UK laws. If you discover content in the Research Portal that you believe breaches copyright or violates any law, please contact openaccess@qub.ac.uk. 


\title{
Real-Time Monitoring of Biopharmaceutical Crystallization: Chord Length Distribution to Crystal Size Distribution for Lysozyme, rHu Insulin and Vitamin B12
}

\author{
Ajinkya Pandit ${ }^{1}$, Venktesh Katkar ${ }^{1}$, Vivek Ranade ${ }^{2}$ and, Rahul Bhambure*1 \\ ${ }^{1}$ Chemical Engineering and Process Development Division, \\ CSIR-National Chemical Laboratory (NCL), Pune, India. \\ ${ }^{2}$ School of Chemistry and Chemical Engineering, Queen's University, Belfast, UK \\ *rs.bhambure@ncl.res.in, Tel. +9120-25902318.
}

\begin{abstract}
:
This investigation is focused on designing an effective strategy for the real-time monitoring of biopharmaceutical crystallization using focused beam reflectance measurement (FBRM). Lysozyme, rHu Insulin and Vitamin B12 were selected as model biopharmaceutical molecules for the study. Under selected experimental conditions, Lysozyme exhibits polymorphism with tetragonal and needle-shaped crystals, rHu Insulin forms rhombohedral crystals, whereas Vitamin B12 forms polyhedral shaped crystals. Focused beam reflectance measurements were performed to obtain the real time chord length distribution (CLD) of crystals obtained using static and dynamic conditions. Mathematical models were developed for converting CLD data obtained using FBRM measurements into the useful crystal size distribution (CSD) data for low (tetragonal, rhombohedral and polyhedral) and high aspect ratio crystals (needle). For high aspect ratio crystals, both width-based and length-based models were developed for capturing the CSD. The model validation was performed by comparing the model CSD with the CSD obtained using microscopic image analysis. Developed models effectively capture the CSD for variable shape crystals of Lysozyme, rHu Insulin and Vitamin B12. It was demonstrated that in case of high aspect ratio crystals, there is an intrinsic limitation in using FBRM for lengthbased crystal size measurement.
\end{abstract}

Keywords: chord length, crystal size, crystal shape, aspect ratio, rHu Insulin, Vitamin B12 


\section{Introduction}

Recently crystallization has gained attention as an effective downstream processing alternative to the existing chromatographic purification of biopharmaceuticals. Crystallization offers numerous advantages in downstream processing of biopharmaceuticals such as - single step cost-effective process, higher product purity and concentration leading to minimal product handling and storage cost, enhanced product shelf life etc. ${ }^{1,2}$ However, protein crystallization has not yet found its way to commercialization in pharmaceutical industries due to intrinsic problems associated with biotherapeutic proteins such as complex molecular structure, high shear and temperature sensitivity, unavailability of generalized criteria for scale up and monitoring of crystallization process. ${ }^{3}$ To facilitate the use of crystallization for downstream processing of biopharmaceuticals, availability of suitable real-time monitoring analytical tools is of paramount importance.

Crystallization crucially affects the properties (crystal shape, crystal size distribution, purity) of the crystallized solid product. These properties explicitly dictate the designing of further unit operations such as settling, filtration and drying and also critically affect the quality of finished

product. ${ }^{2}$ Engineering the morphology of crystals to the desired shape and size can significantly alter the physicochemical properties (bio-availability, flowability, filterability etc.) of the biopharmaceutical drug molecules. Crystal habit is influenced by various internal (purity, liquid occlusion etc.) and external factors (temperature, flow around crystals etc.) In the case of macromolecules like proteins, it's challenging to monitor various internal parameters affecting the crystal growth. Therefore, the monitoring and control of the crystallization processes is essential for obtaining the desired product properties. Real-time tracking of crystallization processes is an inherent requirement for successful implementation of various control strategies and the effective integration of process analytical technologies (PAT) for 
protein crystallization. ${ }^{4,5}$ Coulter counter (CC), ultrasonic attenuation spectroscopy (UAS), laser diffraction (LD), focused beam reflectance measurement (FBRM), and image analysis using different microscopic tools are some of the crystallization monitoring tools currently used for measuring crystal size.$^{6-8}$ Each of these tools has their advantages, disadvantages and biases for crystallization monitoring. ${ }^{9,}, 10$. FBRM is one of the most widely used online monitoring tools for crystal size monitoring and operates on the principle of laser backscattering. ${ }^{11,12}$ The detailed working mechanism of FBRM can be found in the works of Ruf et $a l .{ }^{13}$. The advantages of FBRM includes fast, online and in-situ in-process measurements and the ability to monitor even concentrated slurries making it an ideal tool for monitoring crystallization. ${ }^{14,15}$ However, FBRM has a critical limitation. The output of FBRM is in the form of particle counts and chord length distribution (CLD) which cannot be easily transformed into crystal/particle size distribution (CSD).

In recent years, a lot of work has been done to address this problem of converting CLD into CSD. The research work of Hobbel et al. ${ }^{16}$ was aimed at transforming the CLD into CSD for spherical particles whereas Ruf et al. ${ }^{13}$ considered all possible 2D projections of a template 3D particle for determining the CLD. The algorithm that is followed in the conversion of the CLD to the CSD in works mentioned above as well as the in works that followed is similar. It can be described as - Initially, a single particle (monodisperse system) is considered. According to its geometry, a probability function is derived which gives the CLD for the monodisperse particle system. Using the developed probability function for a polydisperse particle system whose size distribution is known, the theoretical CLD is determined, i.e. a CLD is determined for a known CSD. And in the final step, the determination of CSD from known CLD is considered (inverse problem). Researchers have used various approaches to address the issue of conversion of CLD into CSD for different types of particle systems. Iterative Non-Negative Least Squares algorithm ${ }^{17}$, least square techniques ${ }^{18}$, method of projection onto convex sets ${ }^{19}$, 
determination of CSD from CLD for non-spherical particles with orientation bias ${ }^{20}$, empirical methods relating FBRM measurements to $\mathrm{CSD}^{21}$, estimation of $\mathrm{CSD}$ and aspect ratio for needle-shaped crystals ${ }^{22,23}$ exhibit some of techniques developed for determination of CSD from CLD for particles of various shapes.

More recently Pandit and Ranade ${ }^{24}$ developed a simple tool which was implemented using basic spreadsheeting tools to solve the inverse CLD to CSD problem. For a given CSD, the CLD was derived using an approach similar to Ruf et al. ${ }^{13}$ with the exception that only a circular 2D projection was considered which considerably simplified the involved probability expression. It was also assumed in the mentioned work that the CSD may be adequately represented using a two-parameter standard, normal or log-normal distribution which considerably simplified the inverse problem solution as compared to previous studies.

Despite a significant body of work on FBRM, till date, there are limited reports regarding the use of FBRM for the biopharmaceutical crystallization. Issues such as small crystal size, agglomeration and turbidity become prominent in protein crystallization. In the present investigation, for the first time, we have successfully designed an efficient strategy for the realtime monitoring of biopharmaceutical crystallization using FBRM. The first part of the study was focused on developing a suitable stirred tank reactor for batch crystallization of selected biopharmaceutical molecules. Successful crystallization scale-up was followed by the real-time monitoring of crystallization using FBRM. A two parameter modelling framework developed by Pandit and Ranade ${ }^{24}$, was used for determination of CSD of low aspect ratio crystals like Lysozyme tetragonal crystals or rhombohedral rHu Insulin crystals. For high aspect ratio crystals, two new models were developed to capture width-based and length-based CSDs (described later). A comparative assessment was performed between the width-based, lengthbased and SED-based (Pandit and Ranade) ${ }^{23}$ models. The performance of the developed models were compared with the existing SED-based model to demonstrate the intrinsic 
limitation of using the FBRM for length-based crystal size measurement in case of high aspect ratio crystals. The width-based and SED-based models were then validated for biopharmaceutical particle systems of Lysozyme, rHu Insulin and Vitamin B12. The approach and presented results will be useful for extending applications of FBRM for real-time monitoring of biopharmaceutical crystals.

\section{Experimental Section}

\subsection{Materials:}

Chicken egg white Lysozyme (Cat no. MB098) and Vitamin B-12 (Cat no. MB241) were purchased from HiMedia Labs, India. Sodium acetate anhydrous (Cat no. 1.93244.0251), glacial acetic acid Cat no. 61784325001730) and sodium chloride (Cat no. 1.06404.500) were purchased from Merck, India. All the solutions were prepared in ultrapure (18.2 M 2 ) water obtained using Cascada $^{\mathrm{TM}}$ integrated water purification system (Pall Corporation, USA) and filtered using $0.45 \mu \mathrm{m}$ filter paper before use. Protein concentration was measured in triplicate using Nanodrop ${ }^{\mathrm{TM}}$ (Thermo Scientific, Wilmington, DE) 2000 and Shimadzu UV-1800 spectrophotometer (Shimadzu Corporation, Japan) at $280 \mathrm{~nm}$ with an extinction coefficient of 2.64 $\mathrm{L} \cdot \mathrm{g}^{-1} \cdot \mathrm{cm}^{-1}$ for Lysozyme ${ }^{25}$. Controlled addition of the protein solution to the batch crystallizer was achieved using the STOSS $^{\circledR}$ syringe pump (Shreeyash Electro Medicals, India). An impeller for batch crystallizer was 3D printed in PolyAcryloNitrile.

\subsection{Crystallization Experimental Setup:}

The approach described by Smejkal ${ }^{26}$ was used for designing the batch crystallizer. A jacketed glass vessel (Figure 1A) was constructed with an approximate capacity of $120 \mathrm{~mL}(80 \mathrm{~mL}$ crystallization reaction volume). An impeller as depicted in Figure 1B was 3D printed in PolyAcryloNitrile to achieve minimum shear stress in the fluid. It is important to design and 
operate stirred crystallizers in a way to realise appropriate shear levels in the vessel. Very high local shear or local energy dissipation may adversely influence proteins Very low shear may result in inadequate mixing and suspension. In this work, the approach of Henzler ${ }^{27}$ was implemented to select appropriate shear levels. The impeller speed was selected in such a way that the maximum local energy dissipation per unit mass is about $2.00 \mathrm{~W} / \mathrm{kg}$. The mixing intensity of $2.00 \mathrm{~W} / \mathrm{kg}$ indicates the local maximum energy dissipation for the constructed crystallizer system. Maximum local energy dissipation indicates the maximum hydrodynamic stress on the crystals in the stirred reactor. Smejkal et al. have proved that conventional scaleup criteria did not provide satisfactory results for the protein crystallization process in stirred tanks. However, scale-up based on a constant maximum local energy dissipation proved to be applicable for protein crystallization. ${ }^{26}$ Agitation was achieved using an Eka stirrer (Eka Eurostar 40). The temperature inside the reactor was controlled using WiseCircu temperature bath (Daihan Scientific, Seoul, South Korea). Real-time monitoring of the chord length distribution for crystals was achieved using the FBRM ${ }^{\circledR}$ probe model S400 (Mettler Toledo, Columbus, OH, USA) inserted into the crystallizer in such a way that the probe window was about $45^{\circ}$ to the liquid level. A good mixing in the crystallizer prevented accumulation of the crystals on the window of the FBRM probe. Data acquisition using iC-FBRM software (version 4.2.234 Mettler-Toledo AutoChem, Inc Columbia) was started 1 min after solution was added to the crystallizer. The data points were collected at the interval of 5 seconds until the completion of the crystallization experiment.

\subsection{Lysozyme Crystallization:}

Based on the solubility data for Lysozyme $-\mathrm{NaCl}_{\text {system }}{ }^{28}$ and using crystallization phase diagram in existing literature, ${ }^{29,30}$ three different systems with variable salt concentration were selected for the study (Table 1). Micro-batch crystallization experiments were conducted to 
confirm the crystallization of protein at the selected experimental conditions. Two different solutions were prepared for experimentation. Lysozyme powder was dissolved in acetate buffer $\mathrm{pH}$ containing $1.5 \%$ sodium chloride (Solution A). Further solution A was again subjected to buffer exchange with appropriate buffer using a Sephadex ${ }^{\circledR}$ G-25 prepacked chromatography column to remove any formulation ingredients in the Lysozyme powder. This solution was then centrifuged and filtered through a $0.45 \mu \mathrm{m}$ filter. Protein concentration was measured in triplicate using Nanodrop ${ }^{\mathrm{TM}} 2000$ (Thermo Scientific, Wilmington, DE). Solution B which was an acetate buffer containing sodium chloride in desired concentration (Table 1) was prepared to attain the final Lysozyme and salt system. (i.e. $\mathrm{mg} / \mathrm{mL}$ Lysozyme and \% salt). Solution A was initially added in the designed batch crystallizer. Stirrer and the FBRM probe were then added into the vessel through the vessel lid. The temperature of the reactor was maintained at $20^{\circ} \mathrm{C}$. Solution $\mathrm{B}$ was then added to the reactor using a syringe pump, in 60 minutes. The crystallization experiment was performed under static and dynamic conditions. In case of a static crystallization condition, Lysozyme crystals were allowed grow under in the crystallizer without any agitation. At regular interval crystals were monitored using the image analysis. After the crystals are formed the FBRM measurement was performed with agitation to ensure the solution uniformity in the crystallizer. In case of the dynamic crystallization condition, Lysozyme crystals were allowed to grow with agitation.

\subsection{Cooling Crystallization for Vitamin B12:}

An unseeded cooling crystallization of Vitamin B-12 was carried out using the experimental setup described in Section 2.2. 0.90 gm of purified Vitamin B-12 was dissolved in $25.00 \mathrm{~mL}$ of the ultrapure (18.2 M $\Omega$ ) water and filtered using $0.45 \mu \mathrm{m}$ filter paper before use. Microbatch crystallization experiments were conducted to confirm the crystallization of Vitamin B12 at selected experimental conditions. The filtered saturated solution was kept at $45^{\circ} \mathrm{C}$ for 60 minutes to dissolve any crystals that might have nucleated during filtration. Crystallization of 
Vitamin B-12 was achieved by cooling the solution to $10{ }^{\circ} \mathrm{C}$ with a cooling rate of $1{ }^{\circ} \mathrm{C}$ per minute.

\section{5 rHu Insulin Crystallization}

The purified and in-process biosimilar rHu Insulin samples used in this study were provided by one of the biopharmaceutical industry in India. An unseeded cooling crystallization of rHu Insulin was carried out using the developed experimental setup. At sufficiently high supersaturation crystal nucleation was achieved by cooling the solution to $4{ }^{\circ} \mathrm{C}$.

\subsection{Microscopic Image Analysis}

Offline image analysis was performed while the experiment was running, $1 \mathrm{~mL}$ samples were collected and after centrifuging them for short time $(\sim 30 \mathrm{~s}-60 \mathrm{~s})$, images were taken using optical microscopes at different magnifications, namely, 150X (Carl Zeiss Discovery V20 Stereo), 200X and 400X (Nikon Eclipse E200) and post-processed using ImageJ (Version 1.6.0) software to obtain circular equivalent diameter for tetragonal crystals and rhombohedral crystals of Lysozyme and rHu Insulin respectively. In the case of needle-shaped crystals, length and width were determined using the ImageJ (Version 1.6.0) software. These sets of characteristic lengths were then used to obtain the CSD for the crystals. This CSD obtained is known as CSD obtained from image analysis or experimental CSD. This CSD is then normalised using the total particle count of the distribution. A minimum of 100 particles was measured for each case to determine the CSD. To obtain CSD from the model, the CLD of the instant during which the sample was taken for image analysis is processed through the suitable model depending upon crystal shape and dimension chosen as characterizing length. A normalized model CSD obtained from the model is then compared with earlier obtained normalized experimental CSD. 


\section{Model development and algorithm for crystal size distribution}

The flowchart depicting the model algorithm used for converting the CLD to CSD is shown in Figure 2. In this investigation, the simple iterative method starting from guess CSD is used. As reported by Pandit and Ranade ${ }^{23}$, it is a fair and immensely simplifying assumption that the CSD in most naturally occurring systems may be represented using Normal or Log-Normal distributions. Hence, in the present study, it was assumed that the CSD was represented by a log-normal distribution and was described by the mean and standard deviation of the distribution $(\mu$ and $\sigma)$ by following mathematical formula.

$$
f(x, \mu, \sigma)=\frac{1}{2 x \sigma \sqrt{2 \pi}} e^{-\frac{(\ln x-\mu)^{2}}{\sigma^{2}}}
$$

The model derivation is critically dependent upon the particle shape. Initially, a crystal shape was defined for $2 \mathrm{D}$ representation of the particle. The probability function was then derived for finding the probability of measuring a chord length between two specific lengths (characteristic length scales depending upon the particle shape). This probability function was then used for calculating the chord length distribution for a monodisperse particle system of chosen shape and size. This distribution is discrete distribution defined using bins with upper boundary, lower boundary and a midpoint. In our case, it is 100 bins in the interval of 1 to 1000 $\mu \mathrm{m}$ in such way that the logarithmic spacing between any lower and consecutive upper boundary is the same. The CLD or the CSD depends significantly upon the number of bins chosen. To enable a direct comparison between experimental and simulated CSDs or CLDs, it is imperative that the bin spacing be the same. Hence, the number of bins chosen for the model were the same as those used for FBRM method. The maximum resolution offered by the FRBM method was the bin spacing chosen in the present study. Further, incorporating the same probability function to different single particle systems of various sizes within the required range, a conversion matrix is formulated which contains, the chord length distributions of 
individual particle systems of different sizes. This conversion matrix remains the same if the governing probability equation does not change. Hence for a given system, the conversion matrix remains immutable. Alongside the generation of the conversion matrix, a CSD is defined (using $\mu$ and $\sigma$, lognormal distribution) and normalized using the sum of the entire distribution. This normalized CSD is then converted into a CLD using the predefined conversion matrix. The statistical fit between the obtained CLD and the experimental CLD is observed by calculating the value of the coefficient of determination ' $\mathrm{R}^{2}$ '. If the value of the coefficient of determination is found to be sufficiently close to one, the model proceeds to the next step of comparing the corresponding input CSD for the model to the actual experimental CSD; else, the iterations are continued with different input CSDs until the model CLD fits with the experimental CLD. After comparing the experimental and simulated normalized CSDs for which the corresponding experimental and simulated normalized CLDs were in agreement, if found that both CSDs differ significantly, one may need to revisit the assumptions and check for errors in probability function used to calculate the conversion matrix. Kindly refer to Pandit and Ranade ${ }^{23}$ for a further discussion regarding model development.

Instead of exploring the various projections of a 3D shape, for the solution of the CSD to CLD problem, the present study focused on a rectangular projection of crystals with an aspect ratio characteristic to the system considered. It is hypothesized that such a framework may be used to represent tetragonal systems to even needle-shaped systems as demonstrated in later sections. It was, however, useful to divide the systems into low aspect ratio $(<2)$ and high aspect ratio systems $(>4)$ as it allowed the derivation of simple models for these limiting cases. It is worthwhile to note that it was shown by Pandit and Ranade ${ }^{23}$ that the CSD could be estimated accurately even for tetragonal shaped crystals using a spherical equivalent diameter (SED) model. Thus, it may be inferred that the SED model may prove to be useful for cases of 
low aspect ratio. The following sections outline the derivation of the probability distribution functions using the different modelling approaches for low and high aspect ratio systems.

\subsection{Probability function for low aspect ratio crystals Spherical equivalent diameter (SED) model}

The particle shape in case of the spherical equivalent diameter (SED) model was assumed to be best represented by a circle in 2-D. This assumption transformed the problem of finding the probability that a randomly measured chord chord is between two specific lengths into the problem similar to Bertrand's Paradox. In Bertrand's Paradox, problem considered was to determine the probability that the length of a randomly chosen chord was between two specific lengths on a circle with diameter ' $d$ '. ${ }^{23}$ The end result can be given by the following formula:

$$
P\left(l_{1}, l_{2}, D\right)=\frac{l_{2}^{2}-l_{1}^{2}}{D^{2}}
$$

This formula gives the probability of finding the chords between length $l_{1}$ and $l_{2}$ on a circle of diameter D.

\subsection{Probability function for high aspect ratio crystals}

For high aspect ratio crystals like needles, probability function was derived using two different models. The particle shape for high aspect ratio crystals was assumed to be best represented by a rectangle in 2-D (Figure 3A and 3B). In case of high aspect ratio crystals (where length is more than 8-10 times the width), one may get useful results by assuming that the length is infinite as compared to the width. This assumption is feasible as intuitively one can observe that as the aspect ratio keeps on increasing, the probability of measuring the longest chord (length) keep decreasing drastically (which will be addressed in detail later). However, for 
moderate aspect ratio crystals (length is $2-4$ times the width), the length is still relevant. In the present study, two different approaches were developed to consider these cases as follows:

a. Width-based model

- Length is infinite as compared to the width and hence is not important

- Does not need a specification of aspect ratio

b. Length-based model

- Length is important

- An additional parameter of aspect ratio required to complete system description

The model developed using both these approaches are discussed below.

\section{Width-based model}

The width-based model assumes the width of the rectangle as a smallest chord measured by FBRM (Figure 3A). In case of the width-based model, every chord of a particular length can be uniquely represented by the angle it makes with either vertical or horizontal line passing through the centre (point of intersection of the diagonals) of the rectangle. In the present model, we have considered the angle made with the vertical centre line. If two chords $\mathrm{L}_{1}$ and $\mathrm{L}_{2}$ are considered with lengths $I_{1}$ and $I_{2}$ and the angles they make with vertical centre line be $\theta_{1}$ and $\theta_{2}$, then any chord whose length is between $I_{1}$ and $I_{2}$ must lie in between $\mathrm{L}_{1}$ and $\mathrm{L}_{2}$ when brought to the centre. Thus the angle it makes with the vertical centre line must be between $\theta_{1}$ and $\theta_{2}$. This gives the probability of occurrence of a random chord whose length is between $I_{1}$ and $I_{2}$ by the following equation:

$$
\mathrm{P}\left(\mathrm{l}_{1}, \mathrm{l}_{2}, \mathrm{~W}\right)=\frac{\theta_{1}-\theta_{2}}{\frac{\pi}{2}}
$$

The total sample space for a denominator is $\pi / 2 \operatorname{rad}$ or $90^{\circ}$ due to symmetry that exists across both vertical and horizontal centre lines making all four quadrants essentially identical for 
mathematical treatment. Equation (3), when translated in terms of length $I_{1}$ and $I_{2}$ by use of trigonometry in $\triangle \mathrm{OMA}$ and $\triangle \mathrm{OMB}$ gives us Equation (4)as probability function for a high aspect ratio (needle-shaped) crystal with characteristic dimension as the width of the crystal.

$$
\mathrm{P}\left(\mathrm{l}_{1}, \mathrm{l}_{2}, \mathrm{~W}\right)=\frac{\cos ^{-1}\left(\mathrm{l}_{1} / \mathrm{W}\right)-\cos ^{-1}\left(\mathrm{l}_{2} / \mathrm{W}\right)}{\frac{\pi}{2}}
$$

\section{Length-based model}

In this model, a rectangle of a specific length (whose width is defined by the constant aspect ratio) is considered in the Cartesian plane with its centre at the origin and sides parallel to the axis. The probability function for chord occurrence in this model differs from the previous two models. In this model, a random line was drawn (using random values of slope and intercept) and the length of the chord it cuts on the rectangle was calculated. This step was iterated several times ( thousands) for obtaining a set of chord lengths which are then normalized and transformed into a distribution with pre-specified bins. The number of iterations reflected the particle counts which are typically measured by the FBRM probe in a sample and were usually within a range of 500 to 10,000 . It was assumed that a thousand simulated particles gave a good representation of a simulated CLD measurement. This provides us a chord length distribution for a single particle system. The whole procedure was then repeated for rectangles with different lengths, and the obtained single particle distributions were gathered to form the conversion matrix. This conversion matrix was used as per the model algorithm.

\section{Results and Discussions}

In the present work, we have considered three different models for estimating the crystal size distribution (CSD) given that the chord length distribution (CLD) is known. The first model was the simple model described by Pandit and Ranade ${ }^{23}$ implemented using spreadsheeting tools. The model assumed that the crystals could be adequately represented by spheres and 
provided reasonable estimates for particles with lower aspect ratio crystals (polyhedral, tetragonal etc.) in terms of spherical equivalent diameter (SED). The second was the widthbased model described and developed in the present manuscript which was limited to the case of high aspect ratio (needle shape) crystals. The third model described as the length-based model was also developed in the present manuscript which required the specification of an aspect ratio as a parameter. Initially, a study was performed to compare the predictions provided by the three models for different values of aspect ratios.

The models were then applied for predicting the crystal size distribution and real-time monitoring of Lysozyme, Vitamin B12 and rHu Insulin crystals. The models assume a twoparameter (mean and variance) lognormal distribution function for the normalized CSD. The normalized CLD is derived from the normalized CSD using the developed models. The twoparameter optimization (mean and variance) is carried out such that the error between the experimental and model predicted CLD is minimized. So, as is typical of any optimization problem, initially, guess values are specified for the mean and the variance of the CSD and the values are changed iteratively (the excel solver add-in uses the GRG Nonlinear Solving Method for nonlinear optimization) to improve the fit of the objective function against the target function. In the present case, the normalized model predicted CLD was used as the objective function and the normalized experimental CLD was used as the target function.

\subsection{Evaluation of Models with a Hypothetical Particle System}

A randomly generated particle system with 1000 particles, having a fixed aspect ratio, was considered for model evaluation. The aspect ratio was specified as an input and the particle lengths were generated using a random number generator in MATLAB. The corresponding 
spherically equivalent diameter (SED), and the widths for each of the particles could be calculated from Equation (5). The width was calculated by dividing the length by the aspect ratio and the SED was calculated assuming a 2-dimensional circular cross section that had the same visible area as the rectangle formed using a length and the corresponding width.

$$
\mathrm{D}=\frac{2 \mathrm{l}}{\sqrt{\pi \mathrm{a}}} ; \mathrm{W}=\mathrm{l} / \mathrm{a}
$$

The normalized length-based, width-based and SED-based CSDs were computed. The same bin spacing which is typically used by the FBRM software was used to construct the CSDs to enable a direct comparison across data (100 geometrically spaced bins between 1 to 1000 micron). The three models were then applied to the corresponding CSDs to obtain the model predicted CLDs. The length-based model was developed using the length-based CSD, the spherically equivalent model was developed using the SED-CSD, and the width-based model was developed using the width-based CSD. In the context of calculating the CSD, the widthbased CSD refers to constructing the CSD histogram by considering the particle widths and likewise for length. A comparative assessment between models' performance (SED-based, length-based, width-based) was performed for different aspect ratios. The comparison between CLDs and the corresponding the CSDs obtained using the discussed models for an aspect ratio of one, four and eight are shown in Figures 4 (A, B and C) and 5 (A, B and C) respectively. On comparing the CLDs, it was observed that for low aspect ratios, the length-based model and the SED based model provide equivalent results. As expected, the width-based model predict chords of a larger size than expected. This was due to the intrinsic limitation of the assumption made for deriving the width-based model. The width-based model assumes infinitely long length crystal which was invalid in case of aspect ratio one. Similarly, comparative analysis of CSDs show that the length-based and width-based CSDs overlap for an aspect ratio of one and as expected the SED based CSD is shifted slightly to the right (which can be inferred from the Equation (5)). A comparative analysis was then performed for a 
medium aspect ratio of four. As expected, the CSDs obtained for this case were different for all three models (Figure 5B) but retained their overall shapes. The CLDs predicted using the three models were very similar although the CLD predicted using the SED based model was different than the width and the length-based model. This suggests that as particle aspect ratio increases, the validity of the spherical assumption starts to reduce, and the validity of the needle shape assumption (infinitely long length) starts to take precedence. However, the width-based CLD model was seen to predict larger size chords than the length model which suggested that the length was still relevant for medium aspect ratio crystals. Lastly, a comparison was performed for a high aspect ratio of eight. Like the case of an aspect ratio of 4 , the CSDs for the three cases are significantly different although they have the same shape. The CLDs predicted using the length model, and the width-based models are in good agreement with each other. The CLD predicted using the SED based model is significantly different from the CLDs predicted using the other two models. This was expected and carries forward the trend observed for the case of the aspect ratio of four. Thus it may be concluded that for high aspect ratios (> 4), the length-based model provides equivalent results to the width-based models. From the derivation of the width-based models, we may thus infer that for high aspect ratios $(>4)$ the length of the particle is not relevant. This summarizes a key conclusion as that for high aspect ratio crystals/particles, the FBRM cannot be used to measure the length of the particles, and its use can be only limited to measurement of the width of the crystal/particle. For low aspect ratios $(\sim 1)$, the SED based model agrees well with the length-based model. Hence, for low aspect ratio crystals, the SED based model was used to predict the CSD, and for high aspect ratios (>4) the width-based model was used to predict the CSD in the inverse CLD to CSD problem.

\subsection{Real-time Monitoring of Crystal Size Distribution:}




\subsubsection{Lysozyme}

In case of Lysozyme for system A (30 mg/mL - Lysozyme and $50 \mathrm{mg} / \mathrm{mL}$ - salt, no stirring), tetragonal crystals were obtained as shown in Figure 6A. Similarly, in system B $(40 \mathrm{mg} / \mathrm{mL}-$ Lysozyme, $30 \mathrm{mg} / \mathrm{mL}$ - salt and stirring at $50 \mathrm{rpm}$ ) also tetragonal shaped crystals were obtained as shown in Figure 6B. In experimental system C (30 mg/mL - Lysozyme, $50 \mathrm{mg} / \mathrm{mL}$ - salt and stirring at $125 \mathrm{rpm}$ ), needle-shaped crystals were obtained as shown in Figure 6C. The comparative analysis of experimental and model CLD data and experimental and model CSD data for low aspect tetragonal crystals is shown in Figure 7. It was observed that the model predicts mean crystal size for tetragonal shaped crystals accurately, however, overpredicts the variance. The reason behind this variability may be attributed to observer's bias: During manual microscopic image analysis, the observer measures only unbroken crystals neglecting fines and aggregates using their own discretion leading to a relatively narrow crystal size distribution. However, the FBRM method fails to differentiate between fines, aggregates leading to wide CLD, in turn, giving a relatively wide CSD. A comparison between the expected values of the crystal size diameter (mean diameter) and standard deviations for each of the distributions for various systems are given in Table 2. In the case of Lysozyme tetragonal crystals, the SED model predicts the mean crystal size accurately. The comparative analysis of experimental and model CLD data and experimental and model CSD data for high aspect ratio needle-shaped crystals obtained for the width-based model is shown in Figure 8A and 8B respectively. An excellent agreement between the experimental and model normalized CLD was obtained using the width-based model.

\subsection{2 rHu Insulin:}

A crystalline biopharmaceutical drug, rHu biosimilar Insulin was chosen in this investigation as a second model protein. The observed rhombohedral Insulin crystals are transparent with all 
faces and edges as observed in Figure 9. The CSD was obtained through the image analysis done on a random sample size of 600 crystals. The two parameter values representing the mean and variance of the crystal size distribution were obtained by fitting the normalized CLD obtained using the SED model with the normalized CLD measured using the FBRM probe. A comparative analysis plot for the model fitting is shown in Figure 9. The $\mathrm{R}^{2}$ value for the fit was 0.99 indicating a good agreement between the model CLD and experimental CLD. A comparison between the normalized CSD measured using image analysis and the normalized CSD obtained using the SED model is shown in Figure 9. As seen from this figure, the normalized CSD obtained using the SED model is in good agreement with the CSD obtained using image analysis. This case study successfully demonstrates the use of the SED model for the real-time CSD measurement of low aspect rhombohedral crystals.

\subsubsection{Vitamin-B12}

An unseeded rapid cooling experiment was carried out to achieve the Vitamin B12 crystallization. Polyhedral crystals of Vitamin B12 were obtained as shown in Figure 10 A. The experimental crystal size distribution was estimated through analysis of microscopic images counting of a total of 223 particles. It was observed that matching the model predicted CLD to the experimental CLD led to a significant underprediction of the CSD. This was because the CLD indicated the presence of a substantial number of particles with size less than $10 \mu \mathrm{m}$. However, particles below $10 \mu \mathrm{m}$ could not be measured through image analysis. For such cases, the guess CSD was changed iteratively such that the model predicted squared CLD matched the experimental squared CLD. Squaring the CLD gave a preference to the larger size particles and effectively filtered out the smaller sized particles. A comparison between the experimental and model predicted normalized squared CLDs $\left(R^{2}=0.814\right)$ is shown in Figure 
$10 \mathrm{~B}$, and the corresponding correlation between the experimental and the model CSD is shown in Figure 10C. The model predicted particle size distribution had a mean of $30 \mu m$ with a standard deviation of $27 \mu \mathrm{m}$. The particle size distribution obtained through image analysis had a mean of $49 \mu \mathrm{m}$ and a standard deviation of $30 \mu \mathrm{m}$. It was observed that the model underpredicted the mean particle size but was successful in capturing the variance correctly. As the model applicability was tested for bigger size particles and the shape of the crystals does not change with size, we may conclude that the model is valid for the entire size range. Thus, in principle, the model may be used to obtain useful estimates for the actual particle size during the online and in-situ monitoring of Vitamin B12 crystallization by comparing the unsquared model predicted and experimental CLDs. A comparative analysis between the expected values of the average crystal size and the standard deviations for each of the distributions reported in this study for different systems is given in Table 2. The SED model was seen to predict good results for the case of low aspect ratio crystals. This was encouraging and as expected as the model can be effectively used to predict the crystal size for Lysozyme tetragonal crystals and rhombohedral crystals of rHu Insulin. The width-based model was seen to predict good results for the case of high aspect ratio crystals as was observed for the case of needle-shaped Lysozyme crystals. Both these models were simple excel sheet-based model which did not require the description of any additional parameters and thus would be useful in reliably providing the CSD information without too much work. Further work would be to test the model applicability for various biopharmaceutical systems as also to explore the effect of a possible three-dimensional orientation bias in CLD measurements.

\section{Conclusions}

FBRM coupled with the developed mathematical model can be successfully used as a process analytical technology (PAT) tool for the real-time monitoring of protein crystallization in a 
stirred tank and gain useful insights about the crystal size distribution. This study successfully demonstrated the use of three different models to derive crystal size distribution (CSD) for variable aspect ratio crystals from a chord length measured by FBRM. The model validity was tested for low aspect ratio tetragonal Lysozyme crystals, rhombohedral $\mathrm{rHu}$ Insulin crystals and polyhedral Vitamin B-12 crystals, and similarly for high aspect ratio needle-shaped Lysozyme crystals. In comparison to the existing complex models, the proposed modelling framework offers a simple tool for real-time CSD monitoring for biopharmaceutical crystallization. Even though the model assumes a definite geometric shape for the 2D projections of the crystals like circular or a rectangular (in the case of needle-shaped crystals), it still helps in accurately predicting the crystal size distribution. The reason for this could be attributed to the uniform shaped crystal in selected model systems as opposed to the shape variation which is typically seen in irregularly shaped systems. The tendency of proteins to form precipitate or aggregates is the key challenge for monitoring protein crystallization using FBRM. Despite these shortcomings, it can be concluded that the FBRM probe can be effectively used to monitor mean crystal size. The developed model underpredicts the spread of crystal size distribution, the reason for which can be attributed to the presence of fine precipitate/aggregate. On another note, the back-scattering of the laser from the FBRM is influenced by a number of crystal properties ${ }^{31,32}$ (such as the refractive index difference between crystals and the liquid, opacity of crystals etc.). Particle systems where the backscattering behavior of the laser is non-ideal, present an inherent limitation for the FBRM technique itself. Hence, it is always a good idea to perform a validation study for the system considered before using the model. It was also demonstrated that in case of high aspect ratio crystals, there is an intrinsic limitation in using FBRM for length-based crystal size measurement. For a successful crystal engineering, real-time monitoring of the crystal size and shape distribution is a crucial necessity. In future, it is conceivable that effective integration of 
the developed modelling platform with crystallizer can help in successful crystal engineering of biopharmaceutical crystals.

\section{Symbols and Notations}

\begin{tabular}{|c|c|c|}
\hline Symbol & Description & Unit \\
\hline$f\left(x, \mu, \sigma^{2}\right)$ & Probability distribution function with mean $\mu$ and variance $\sigma^{2}$ & $\mathrm{~m}^{-1}$ \\
\hline$x$ & Particle size co-ordinate & $\mathrm{m}$ \\
\hline$\mu$ & Mean for probability distribution function & $\mathrm{m}$ \\
\hline$\sigma^{2}$ & Lariance for probability distribution function & $\mathrm{m}^{2}$ \\
\hline$l$ & The diameter of the circular 2D projection of the spherical & $\mathrm{m}$ \\
\hline$D$ & particle & $\mathrm{m}$ \\
\hline \multirow{2}{*}{$\theta$} & The angle which a hypothetically measured chord makes with & $\mathrm{rad}$ \\
\hline & the vertical & $\mathrm{m}$ \\
\hline
\end{tabular}




\begin{tabular}{|l|c|c|}
\hline$a$ & Aspect Ratio of a Crystal & \\
\hline
\end{tabular}

\section{Acknowledgement:}

Authors are grateful to financial support for this work by CSIR-India through MLP031426 project. Ajinkya Pandit and Venktesh Katkar contributed equally to this work. 


\section{References}

(1) Fujiwara, M.; Nagy, Z. K.; Chew, J. W.; Braatz, R. D. First-principles and direct design approaches for the control of pharmaceutical crystallization. J. Process Control 2005, 15, 493.

(2) Nagy, Z. K.; Fevotte, G.; Kramer, H.; Simon, L. L. Recent advances in the monitoring, modelling and control of crystallization systems. Chem. Eng. Res. Des. 2013, 91, 1903.

(3) Schimdt, S.; Havekost, D.; Kaiser, K.; Henzler, H-J. Crystallization for the downstream processing of proteins. Eng. Life Sci. 2005, 5, 273.

(4) Mougin, P.; Thomas, A.; Wilkinson, D.; White, G.; Roberts, K. J.; Herrmann, N.; Jack. R.; Tweedie R. On-line monitoring of a crystallization process. AIChE J. 2003, 49,373 .

(5) Simon, L. L.; Pataki, H.; Marosi, G.; Meemken, F.; Hungerbuhler, K.; Baiker, A.; Tummala, S.; Glennon, B.; Kuentz, M.; Steele, G.; Kramer, H. J. M.; Rydzak, J. W.; Chen, Z.; Morris, J.; Kjell, F.; Singh, R.; Gani, R.; Gernaey, K. V.; Louhi-Kultanen, M.; O'Reilly, J.; Sandler, N.; Antikainen, O.; Yliruusi, J.; Frohberg, P.; Ulrich, J.; Braatz, R. D.; Leyssens, T., Stosch, M.; Oliveira, R.; Tan, R. B. H.; Wu, H.; Khan, M.; O'Grady, D.; Pandey, A.; Westra, R.; Delle-Case, E.; Pape, D.; Angelosante, D.; Maret, Y.; Steiger, O.; Lenner, M.; Abbou-Oucherif, K.; Nagy, Z. K.; Litster, J. D.; Kamaraju, V. K.; Chiu, M.-S. Assessment of recent process analytical technology (PAT) trends: A multiauthor review. Org. Process Res. Dev. 2015, 19, 3.

(6) Li, M.; Wilkinson, D. Determination of non-spherical particle size distribution from chord length measurements. Part 1: Theoretical analysis. Chem. Eng. Sci. 2005, 60, 3251. 
(7) Arnaout, T. EI.; Kurki, L.; Vaarala, T.; Ojala, K.; Cullen, P. J.; Sullivan, C. Crystallization monitoring using simultaneous bright field and PlasDIC imaging. Chem. Eng. J. 2016, 300, 64.

(8) Mikol, V.; Hirsch, E.; Giege, R. Monitoring protein crystallization by dynamic light scattering. FEBS Lett. 1989, 258, 63.

(9) Albuquerque, I.; Mazzotti, M.; Ochsenbein, D. R.; Morari, M. Effect of needle-like crystal shape on measured particle size distributions. AIChE J. 2016, 62, 2974.

(10) Silva, A. F. T.; Burggraeve, A.; Denon, Q.; Meeren, P. V.; Sandler, N.; Kerkhof, T. V. D.; Hellings, M.; Vervaet, C.; Remon. J. P.; Lopes, J. A.; Beer, T. D. Particle sizing measurements in pharmaceutical applications: Comparison of in-process methods versus off-line methods. Eur. J. Pharm. Biopharm. 2013, 85, 1006.

(11) Heinrich, J.; Ulrich, J. Application of laser-backscattering instruments for in situ monitoring of crystallization processes - a review. Chem. Eng. Technol. 2012, 35, 967.

(12) Chew, J. W.; Chow, P. S.; Tan, R. B. H. Automated in-line technique using FBRM to achieve consistent product quality in cooling crystallization. Cryst. Growth Des. 2007, $7,1416$.

(13) Ruf, A.; Worlitschek, J.; Mazzotti, M. Modeling and experimental analysis of CSD measurements through FBRM. Part. Part. Syst. Charact. 2000, 17, 167.

(14) Leyssens, T.; Baudry, C.; Hernandez, M. L. E. Optimization of a crystallization by online FBRM analysis of needle-shaped crystals. Org. Process Res. Dev. 2011, 15, 413.

(15) Heath, A. R.; Fawell, P. D.; Bahri, P. A.; Swift, J. D. Estimating average particle size by focused beam reflectance measurement (FBRM). Part. Part. Syst. Charact. 2002, 19,84

(16) Hobbel, E. F.; Davies, R.; Rennie, F. W.; Allen, T.; Butler, L. E.; Waters, E. R.; Smith, J. T.; Sylvester, R. W. Modern methods of on-line size analysis for particulate process streams. Part. Part. Syst. Charact. 1991, 8, 29.

(17) Li, M.; Wilkinson, D.; Patchigolla, K. Comparison of particle size distributions measured using different techniques. Part. Sci. Technol. 2005, 23, 265.

(18) Bloemen, H. H. J.; De Kroon, M. G. M. Transformation of chord length distributions into particle size distributions using least squares techniques. Part. Sci. Technol. 2005, 23, 377. 
(19) Worlitschek, J.; Hocker, T.; Mazzotti, M. Restoration of PSD from chord length distribution data using the method of projections onto convex sets. Part. Part. Syst. Charact. 2005, 22, 81.

(20) Nere, N. K.; Ramkrishna, D.; Parker, B. E.; Bell, W. V.; Mohan, P. Transformation of the chord-length distributions to size distributions for nonspherical particles with orientation bias. Ind. Eng. Chem. Res. 2007, 46, 3041.

(21) Li, H.; Grover, M. A.; Kawajiri, Y.; Rousseau, R. W. Development of an empirical method relating crystal size distributions and FBRM measurements. Chem. Eng. Sci. 2013, 89, 142.

(22) Agimelen, O. S.; Hamilton, P.; Haley, I.; Nordon, A.; Vasile, M.; Sefcik, J.; Mulholland, A. J. Estimation of particle size distribution and aspect ratio of nonspherical particles from chord length distribution. Chem. Eng. Sci. 2015, 123, 629.

(23) Agimelen, O. S.; Jawor-Baczynska, A.; McGinty, J.; Dziewierz, J.; Tachtatzis, C.; Cleary, A.; Haley, I.; Michie, C.; Andonovic, I.; Sefcik, J.; Mulholland, A. J. Integration of in situ imaging and chord length distribution measurements for estimation of particle size and shape. Chem. Eng. Sci. 2016, 144, 87.

(24) Pandit, A. V.; Ranade, V. V. Chord length distribution to particle size distribution. AIChE J. 2016, 62, 4215.

(25) Sophianopoulos, A. J.; Rhodes, C. K.; Holcomb, D. N.; Van Holde, K. E. Physical studies of Lysozyme. J. Biol. Chem. 1961, 237, 1107.

(26) Smejkal, B.; Helk, B.; Rondeau, J. M.; Anton, S.; Wilke, A.; Scheyerer, P.; Fries, J.; Hekmat, D.; Weuster-Botz, D. Protein crystallization in stirred systems - scale-up via the maximum local energy dissipation. Biotechnol. Bioeng. 2013, 110, 1956.

(27) Henzler, H-J. Particle stress in bioreactors. In: Influence of Stress on Cell Growth and Product Formation. Advances in Biochemical Engineering/Biotechnology, Schugerl K.; Kretzmer, G.; Henzler H-J.; Kieran P. M.; MacLoughlin P. E.; Malone D. M.; Schumann, W.; Shamlou, P. A.; Yim, S. S.; Eds.; Springer, Berlin, Heidelberg, 2000; pp 35-82.

(28) Howard, S. B.; Twigg, P. J.; Baird, J. K.; Meehan, E. J. The solubility of hen egg white lysozyme. J. Cryst. Growth 1988, 90, 94.

(29) Groß, M.; Kind, M. Bulk crystallization of proteins by low-pressure water evaporation. Chem. Eng. Technol. 2015, 39, 1483. 
(30) Castro, F.; Ferreira, A.; Teixeira, J. A.; Rocha, F. Protein crystallization as a process step in a novel meso oscillatory flow reactor: study of lysozyme phase behavior. Cryst. Growth Des. 2016, 16, 3748.

(31) Kee, N. C. S.; Woo, X. Y.; Goh, L. M.; Rusli, E.; He, G.; Bhamidi, V.; Tan, R. B. H.; Kenis, P. J. A.; Zukoski, C. F.; Braatz, R. D. Design of crystallization processes from laboratory research and development to the manufacturing scale. Part I Am Pharmaceut Rev 2008, 11, 110.

(32) Kee, N. C. S.; Woo, X. Y.; Goh, L. M.; Rusli, E.; He, G.; Bhamidi, V.; Tan, R. B. H.; Kenis, P. J. A.; Zukoski, C. F.; Braatz, R. D. Design of crystallization processes from laboratory research and development to the manufacturing scale. Part II $A m$ Pharmaceut Rev 2008, 11, 74.

\section{Figures captions}

Figure 1: Batch crystallization vessel used for the experimentation. A - schematic diagram of crystallizer used for the experimentation, B - experimental crystallization set-up, C- 3D printed impeller design used for the experimentation

Figure 2: Algorithm for the mathematical model used for converting the chord length distribution to crystal size distribution

Figure 3: 2D projection of a needle-shaped crystal. A-width-based model B-length-based model

Figure 4: Comparative analysis between the model predicted CLDs obtained using the SED, width and length-based models for an aspect ratio of one (A) four (B) and eight (C).

Figure 5: Comparative analysis between the normalized CSDs for an aspect ratio of ratio of one (A) four (B) and eight (C) using SED, width and length-based model.

Figure 6: Microscopic image analysis of Lysozyme crystals. A - static crystallization forming tetragonal crystals (at 400X magnification), B - dynamic crystallization forming tetragonal crystals (at 400X magnification), C - dynamic crystallization forming needle-shaped crystals (at 150X magnification)

Figure 7: Use of SED model to monitor the low aspect ratio tetragonal shaped Lysozyme crystals. A - Comparison between CLD obtained from FBRM and CLD determined using mathematical model under static experimental condition. B - Comparison between CLD obtained from FBRM and CLD determined using mathematical model under dynamic experimental condition. $\mathrm{C}$ - Comparison between CSD obtained from image analysis and CSD determined using a mathematical model for the dynamic experimental state. 
Figure 8: Use of the width and length-based model to monitor the high aspect ratio needleshaped Lysozyme crystals. A - Comparison between CLD obtained from FBRM and CLD determined using mathematical model B - Comparison between CSD obtained from image analysis and CSD determined using a mathematical model. C - Comparison between CSD obtained from image analysis and CSD determined using a mathematical model.

Figure 9: Use of SED model to monitor the rhombohedral shaped rHu Insulin crystals. A: Microscopic image analysis of rHu Insulin crystals. A - Comparison between CLD obtained from FBRM and CLD determined using mathematical model B - Comparison between CSD obtained from image analysis and CSD determined using a mathematical model.

Figure 10: Use of SED model to monitor the polyhedral shaped Vitamin B12 crystals. A: Microscopic image analysis of Vitamin B12 crystals. B - Comparison between CLD obtained from FBRM and CLD determined using mathematical model C - Comparison between CSD obtained from image analysis and CSD determined using a mathematical model. 
Figure 1

FBRM Probe 


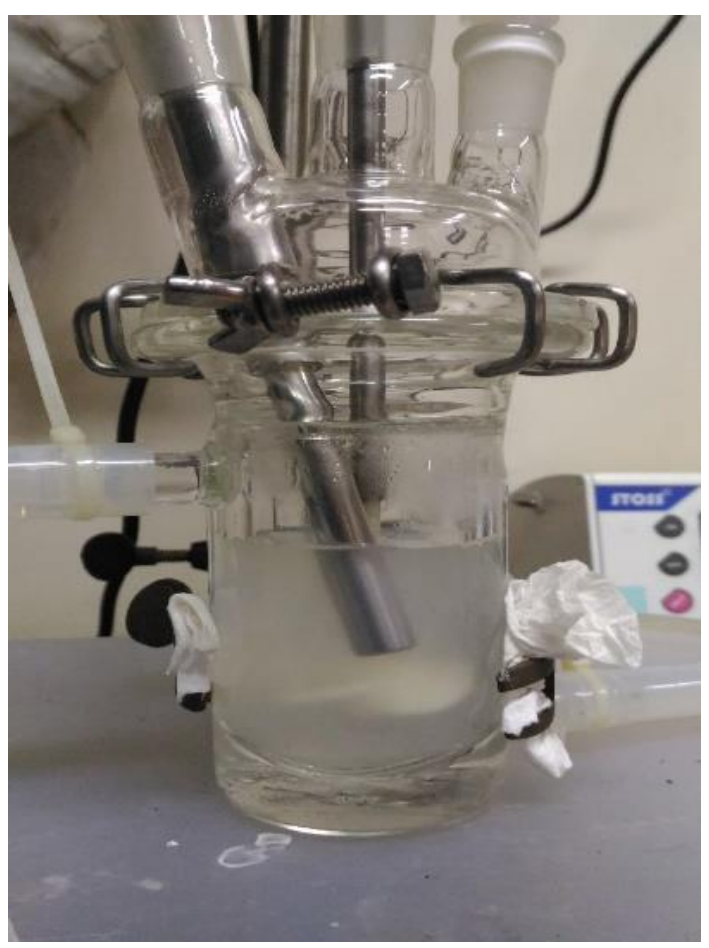

C 
Figure 2

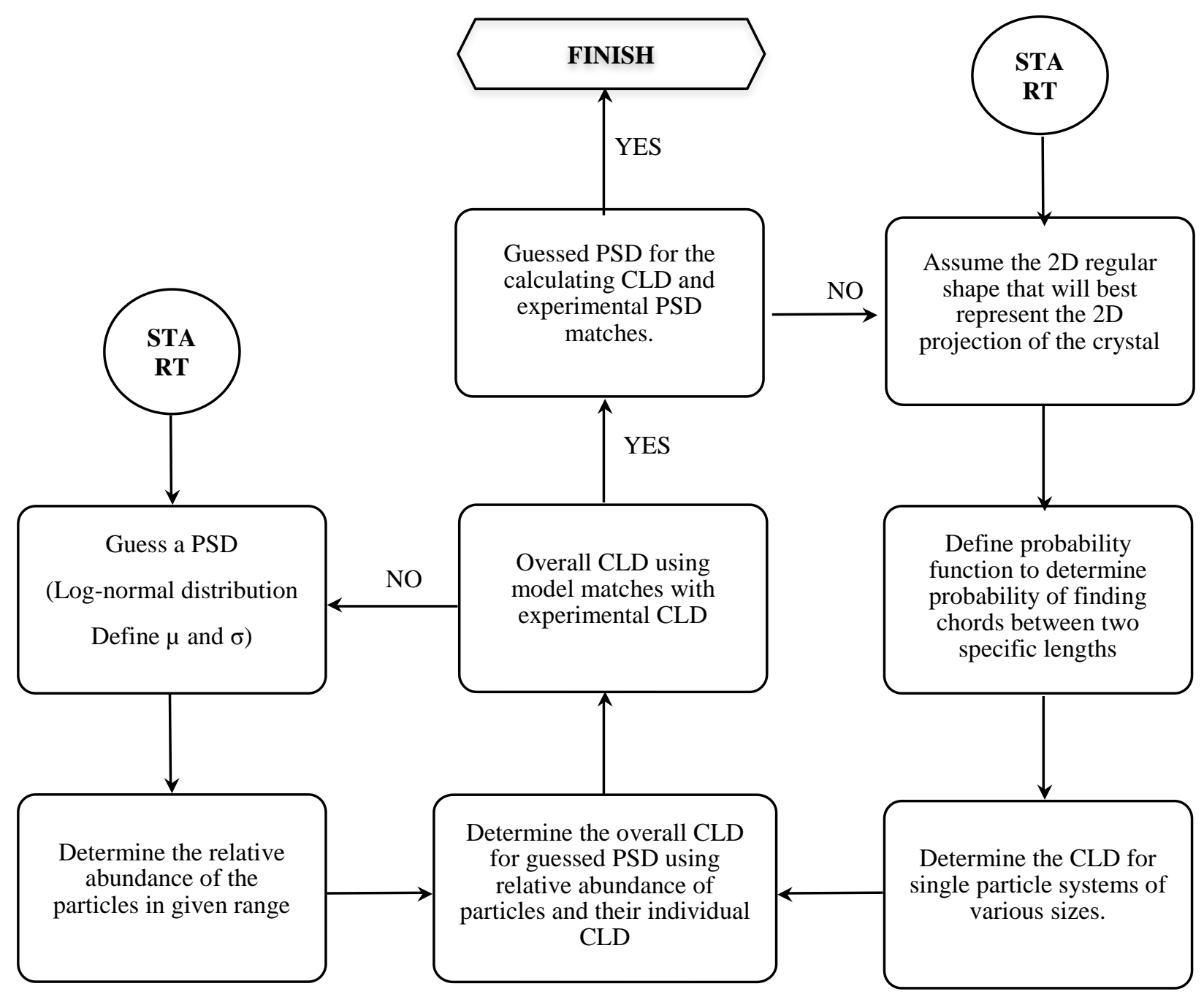


Figure 3

A

$\mathrm{L}$ - Length of needle

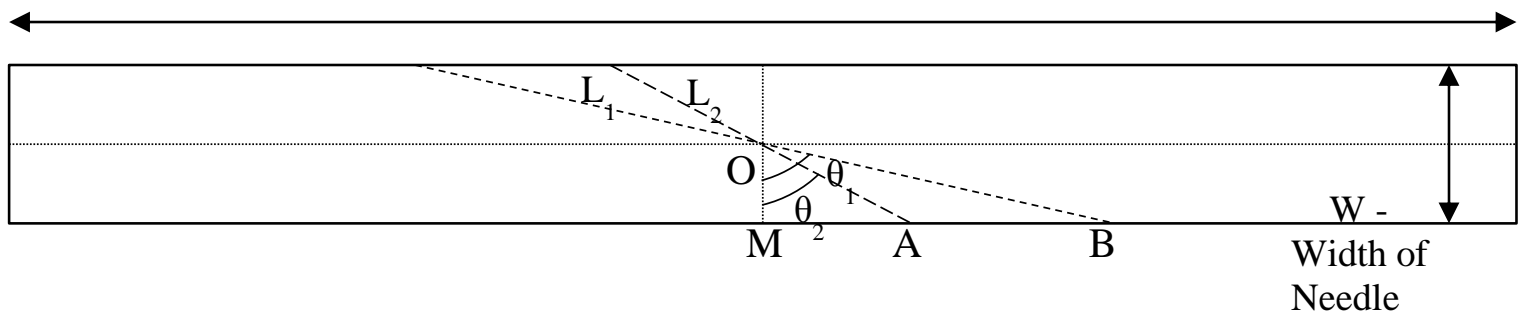

B

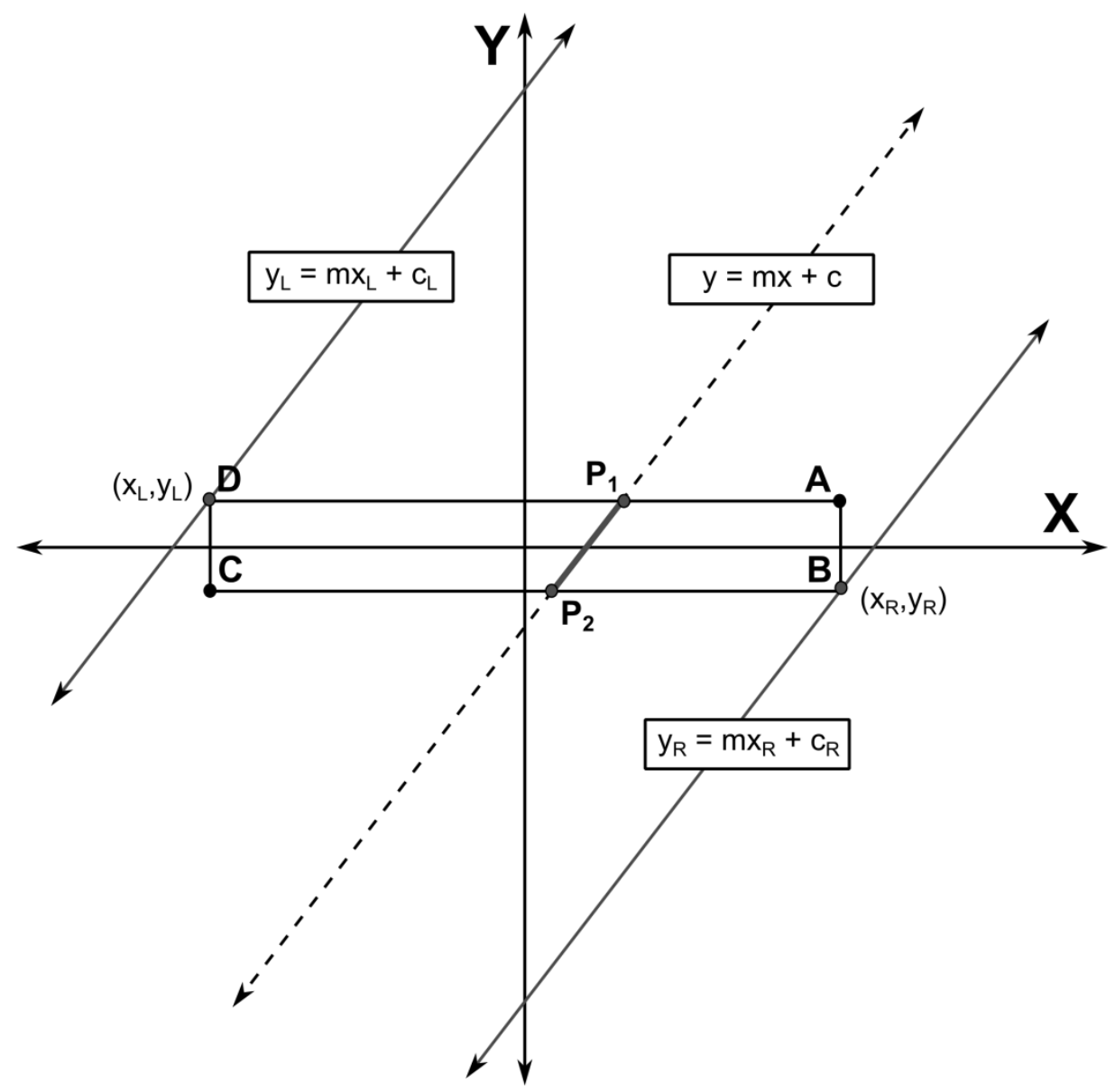


Figure 4

4(A)

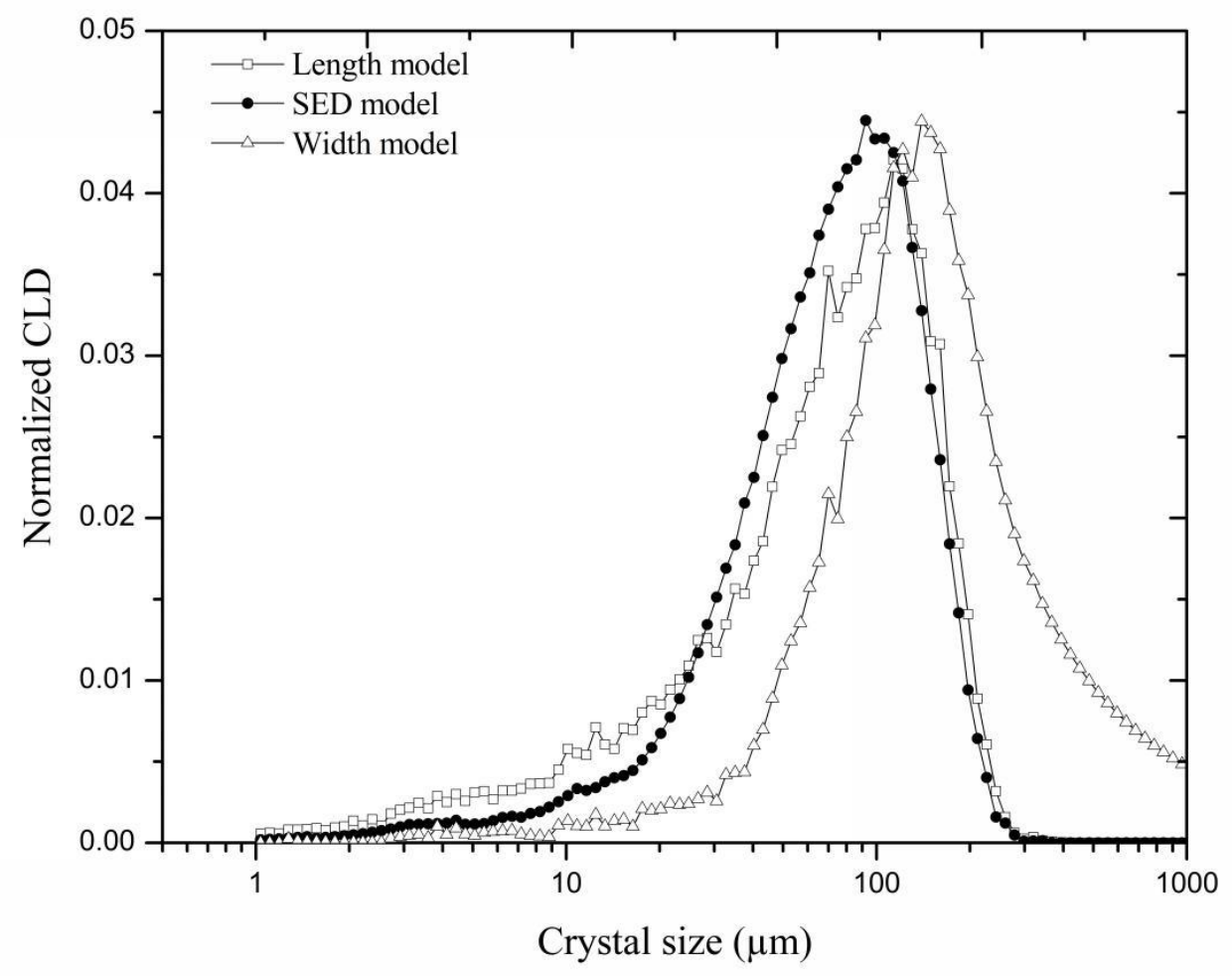




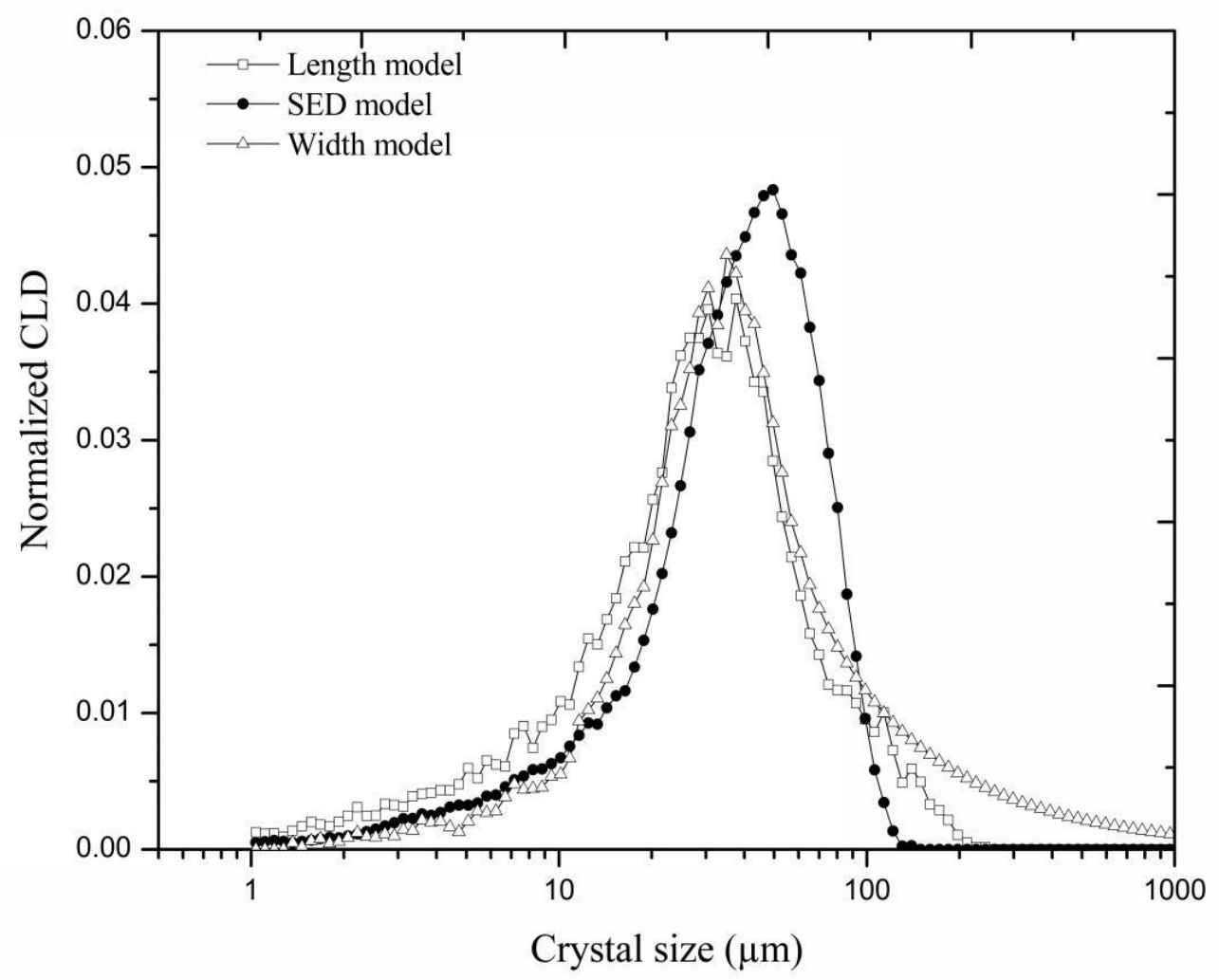


4(C)

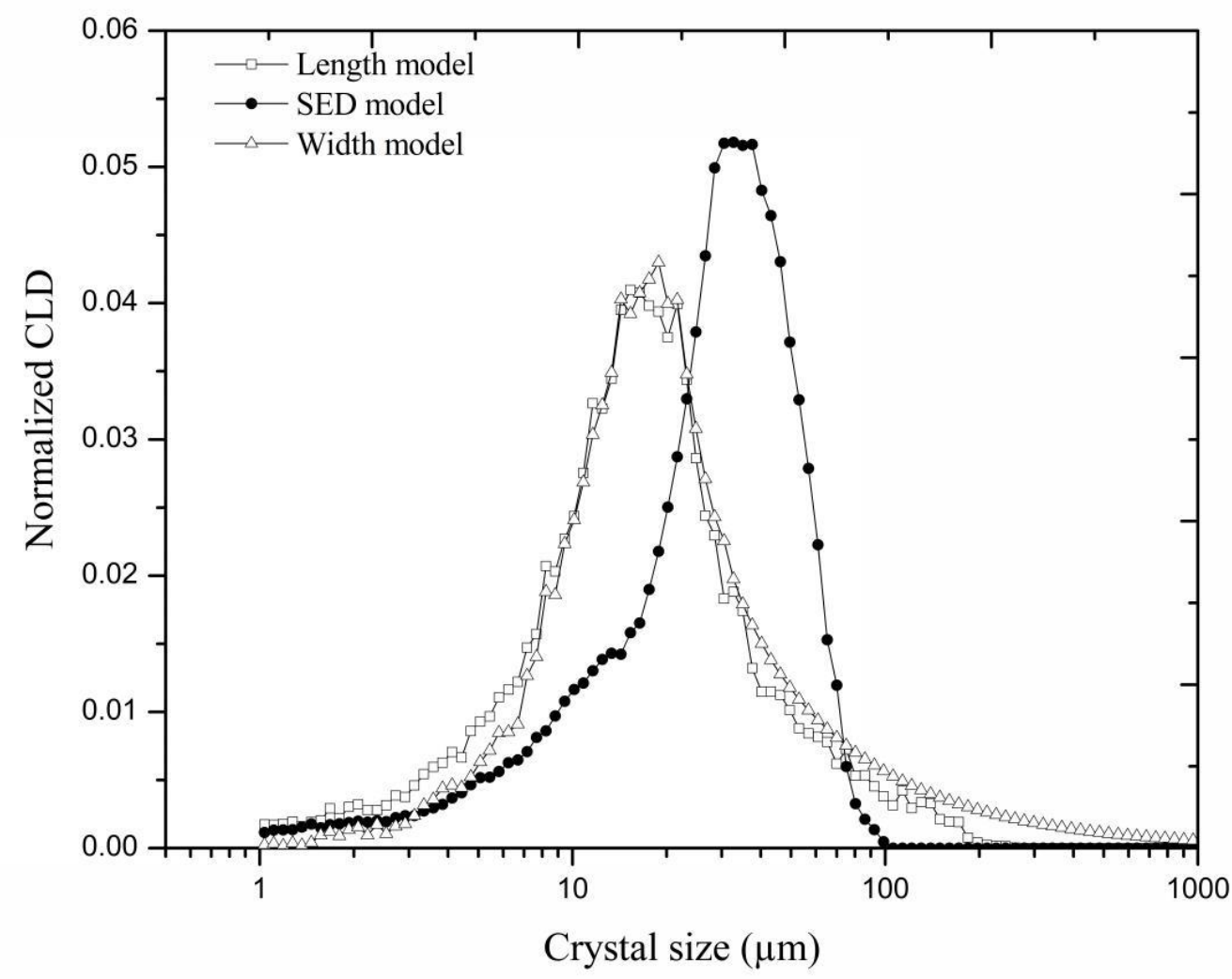


Figure 5

5(A)

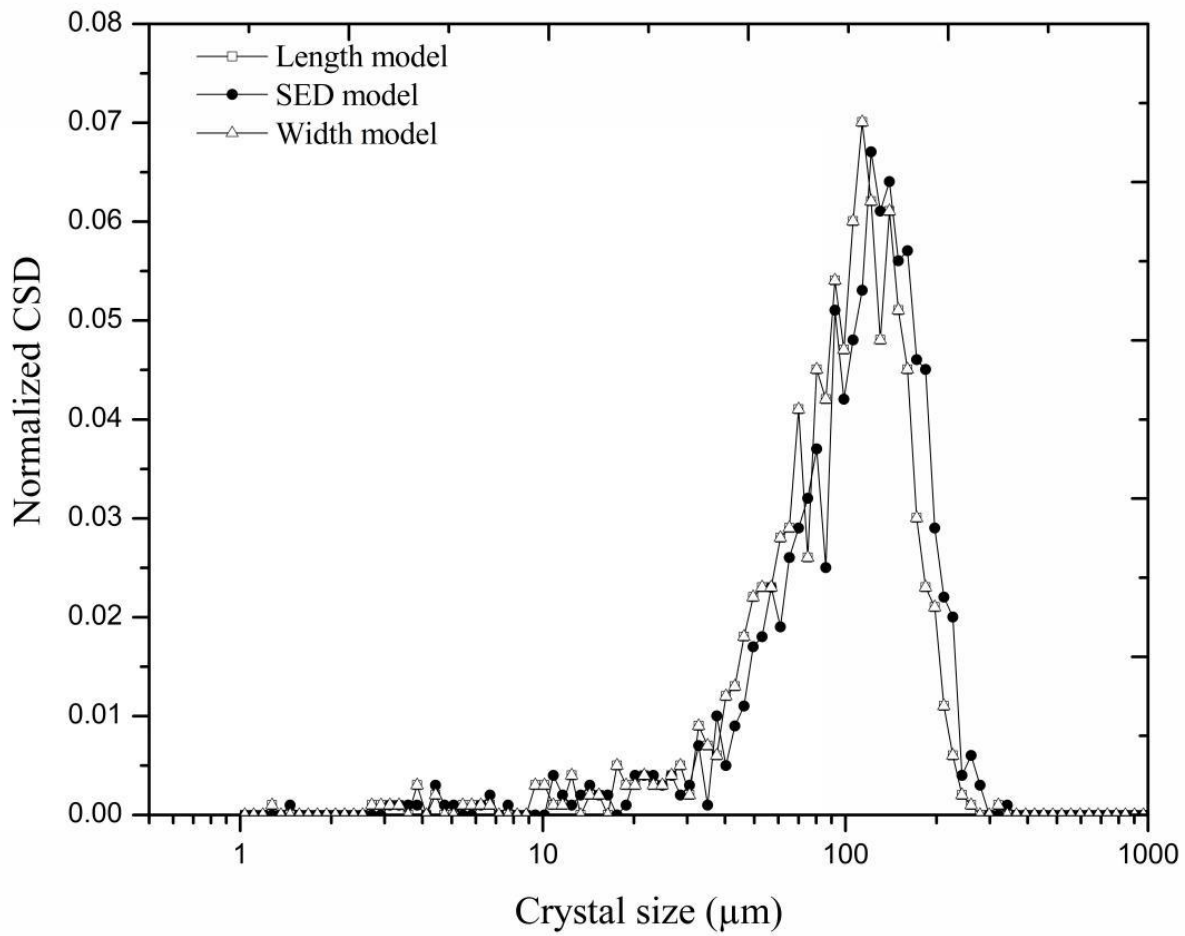




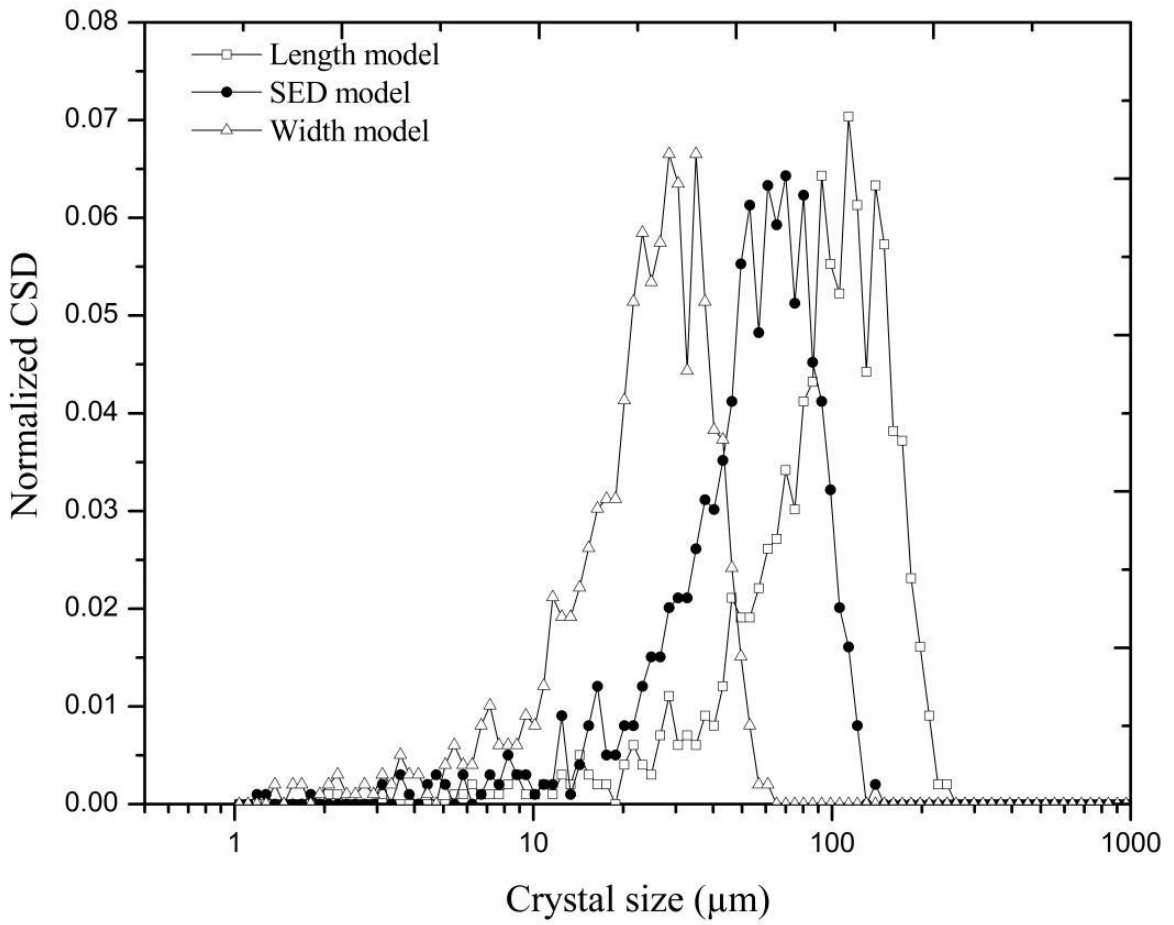


5(C)

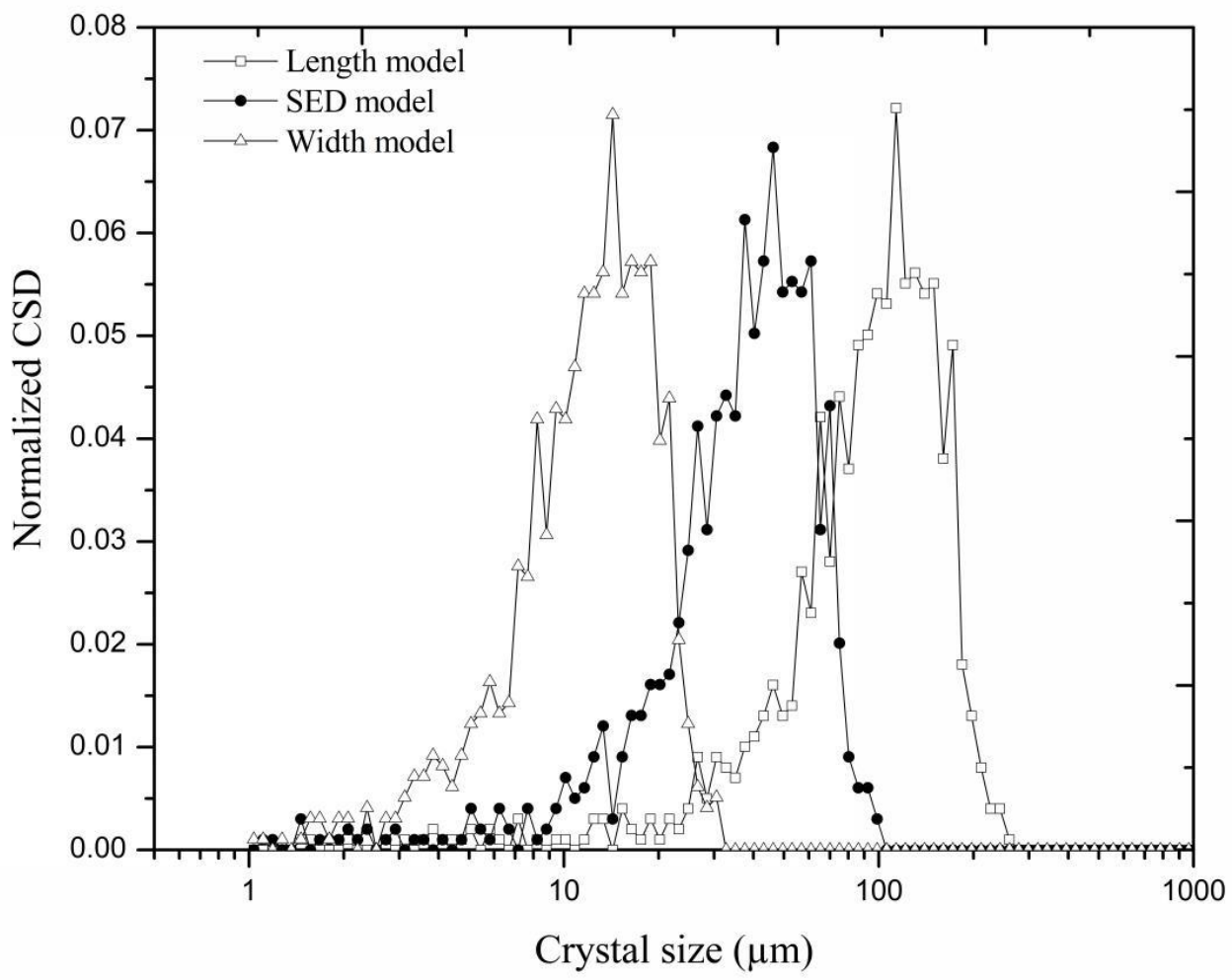


Figure 6

6(A)

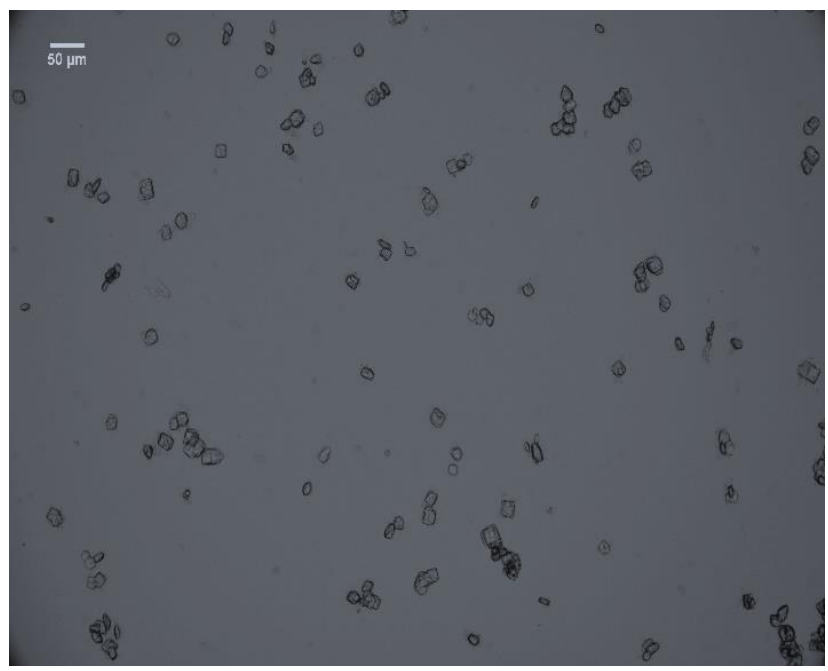

6(B)

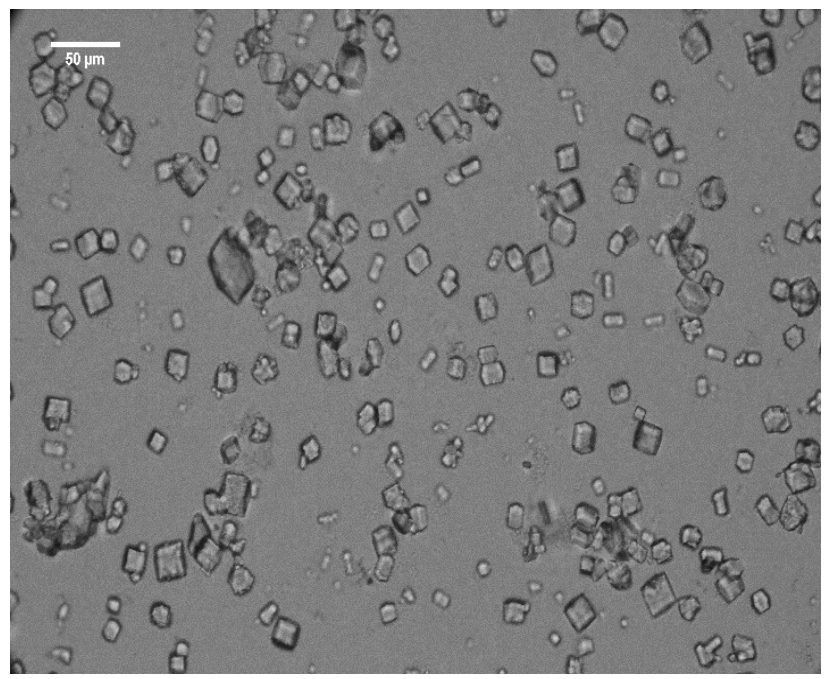

6(C)

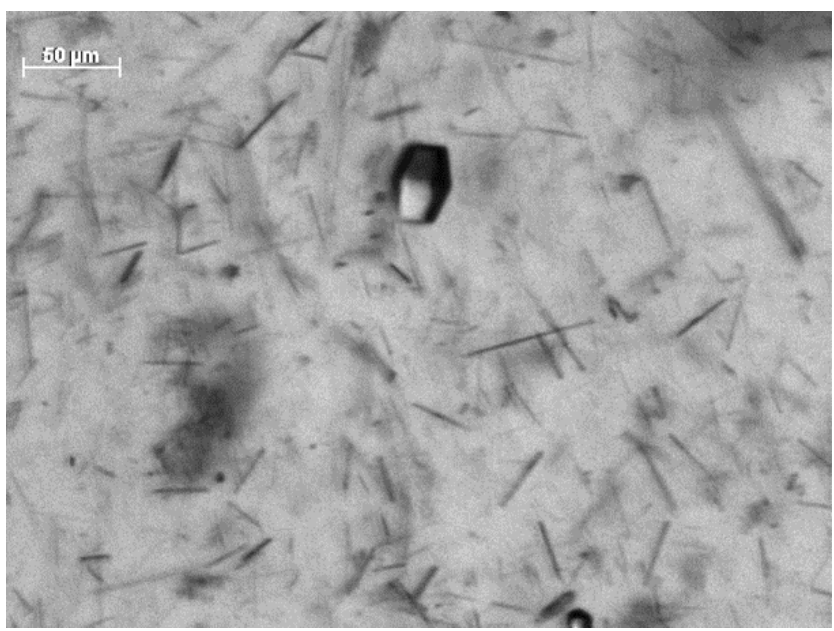


Figure 7

7(A)

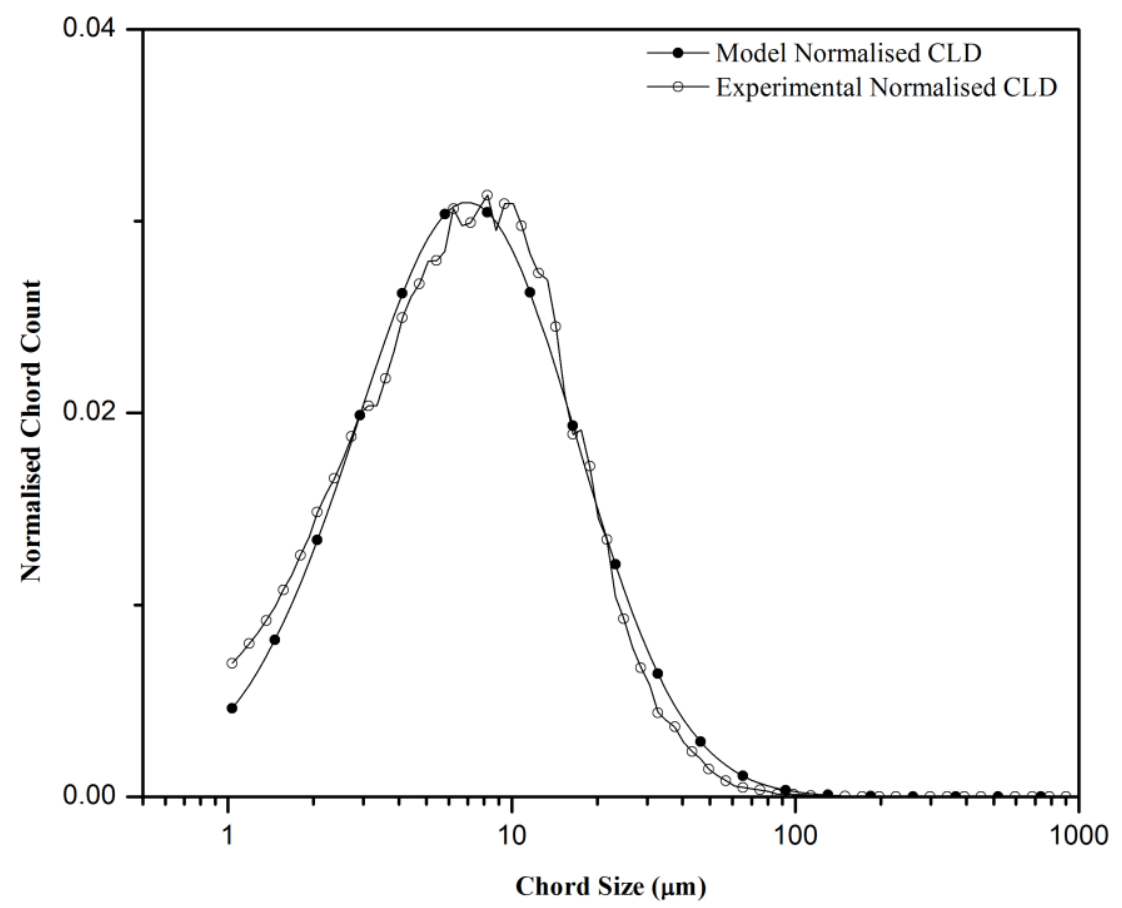

7(B)

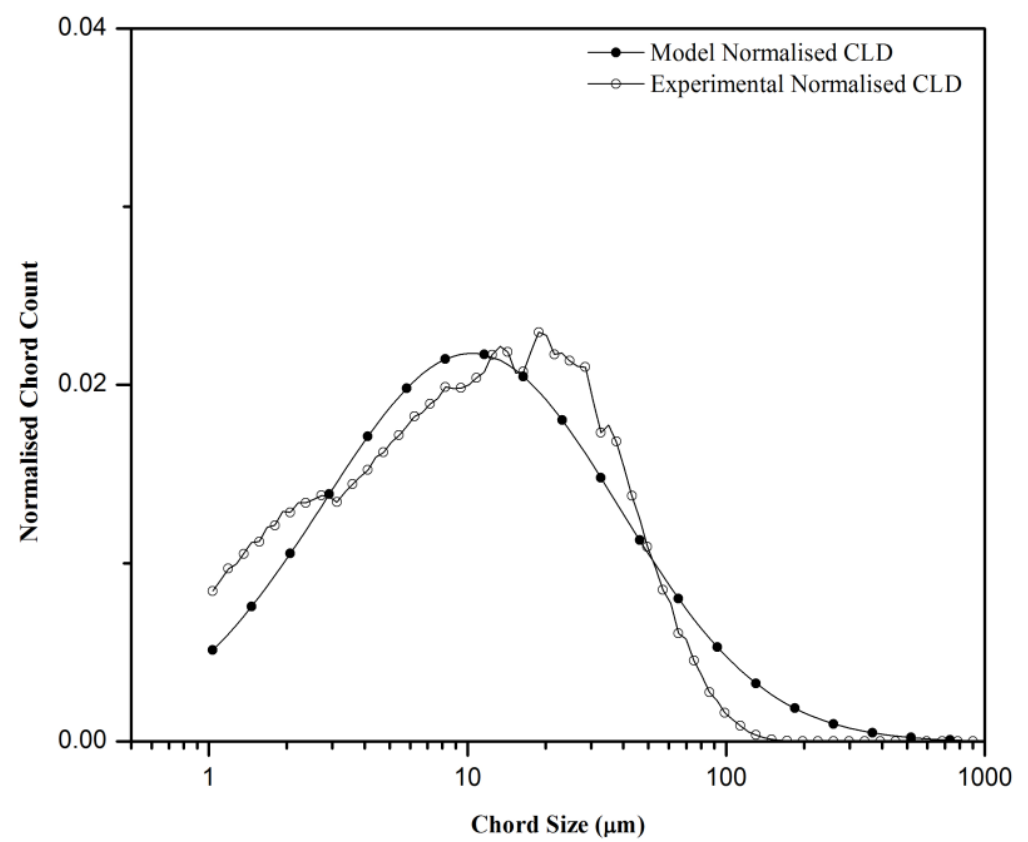




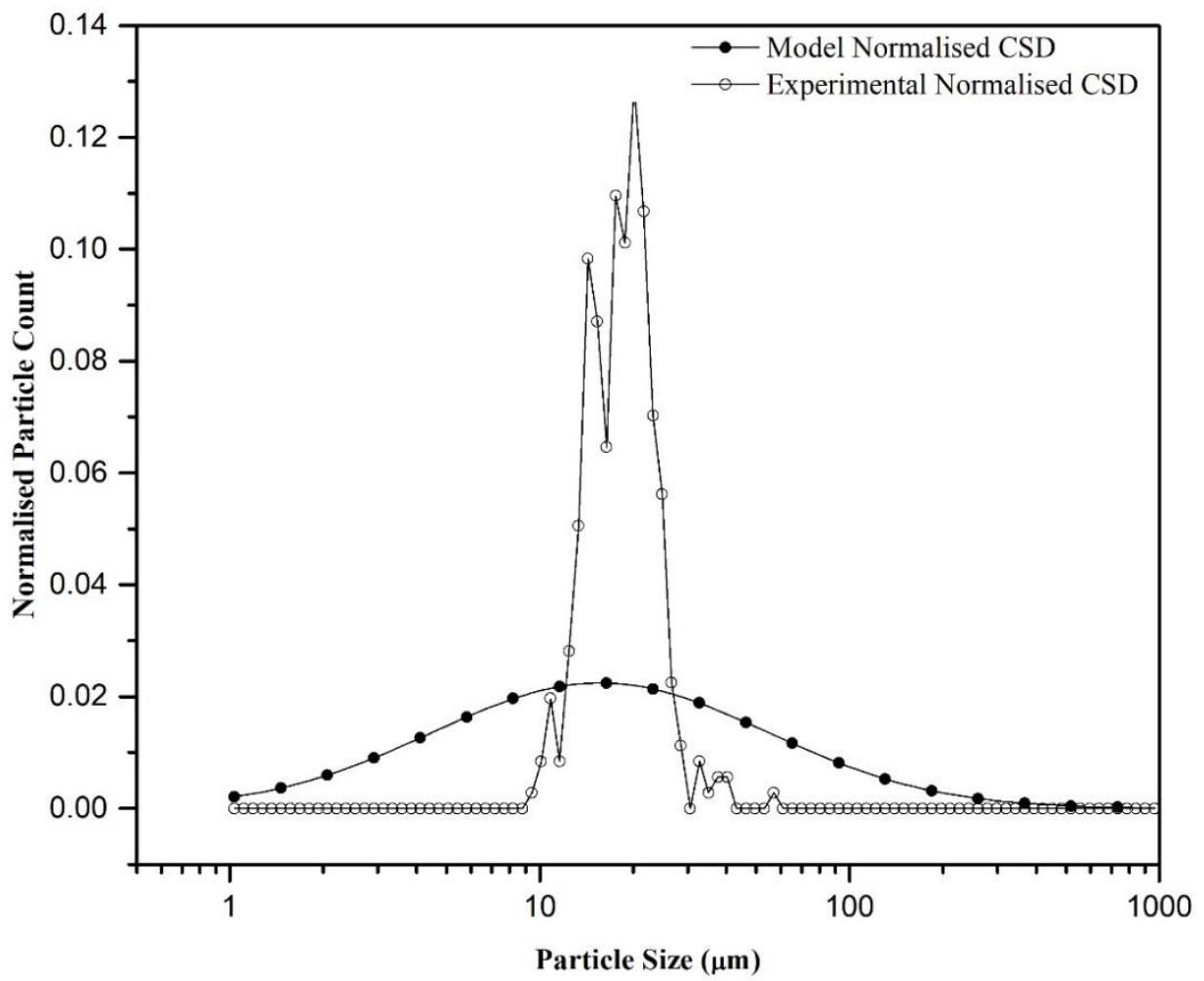




\section{Figure 8}

8(A)

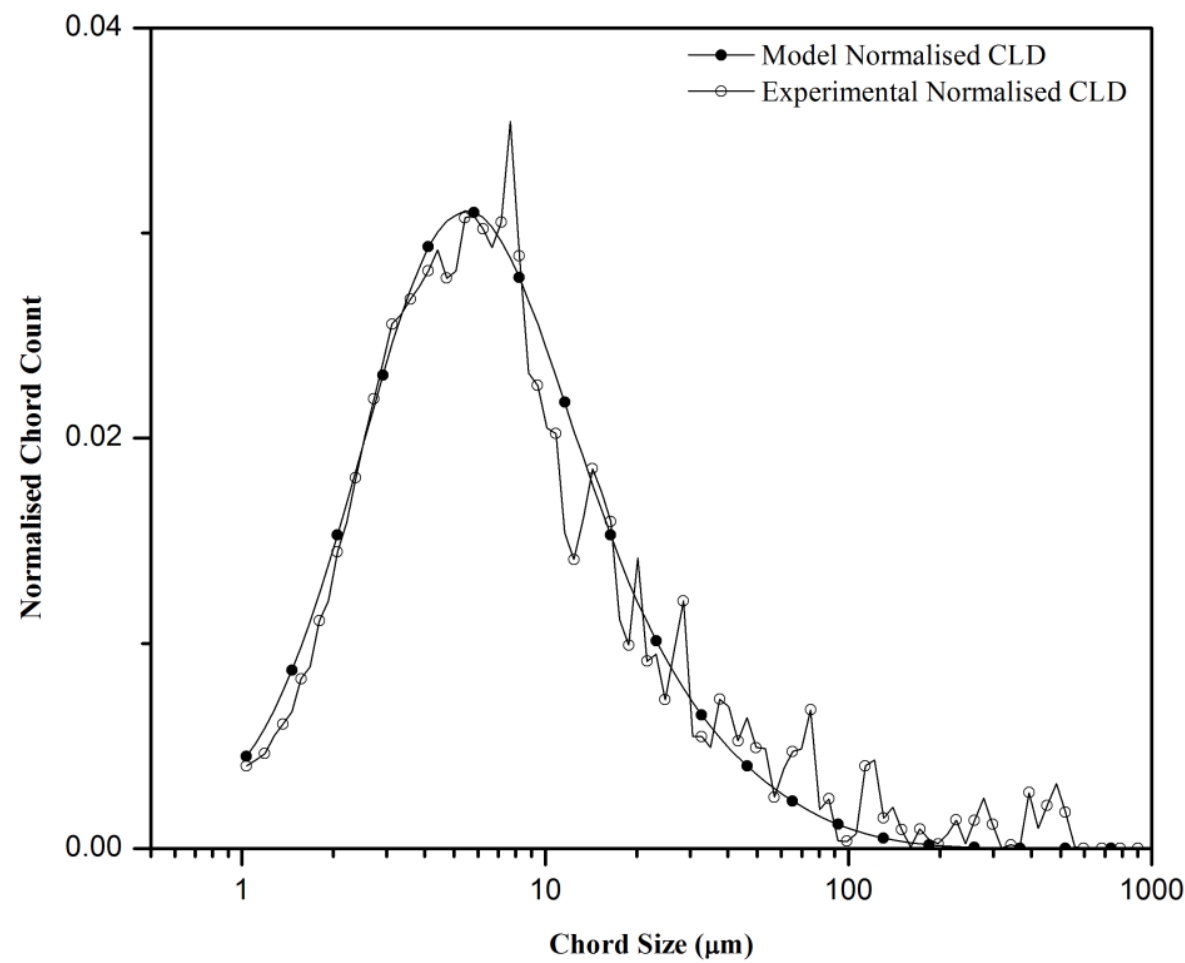

8(B)

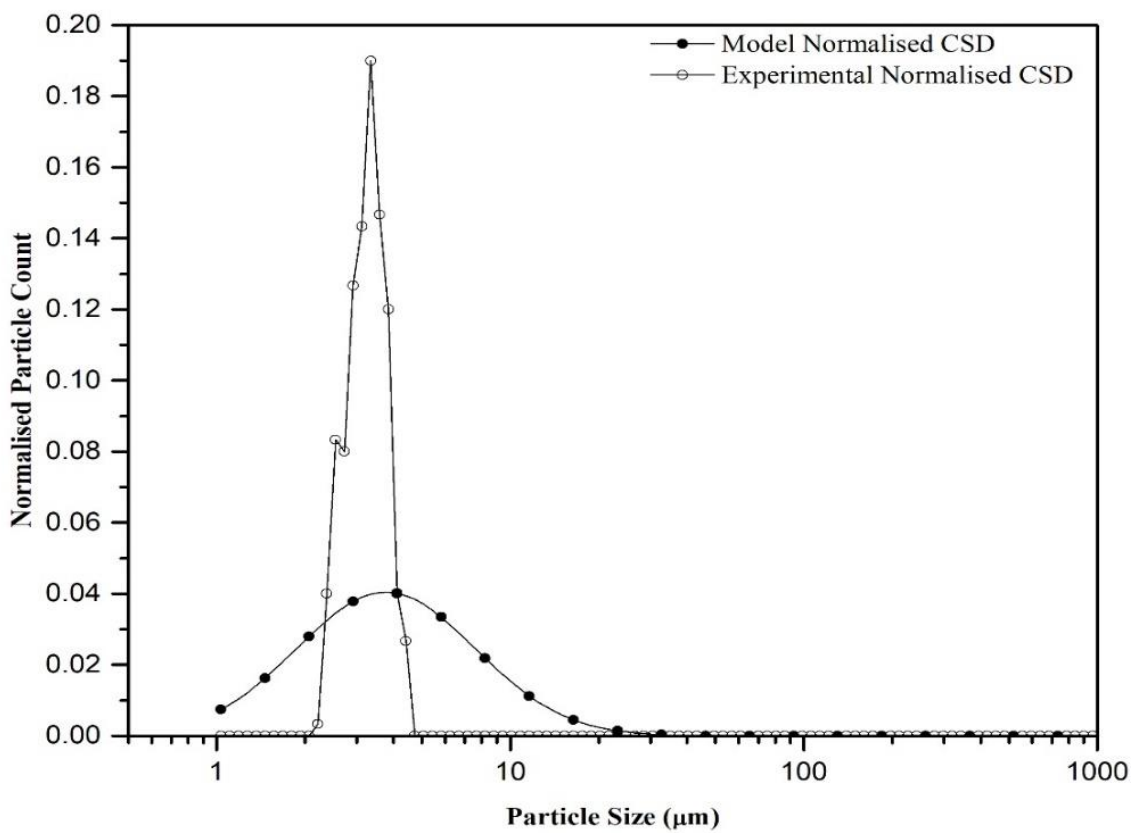


8(C)

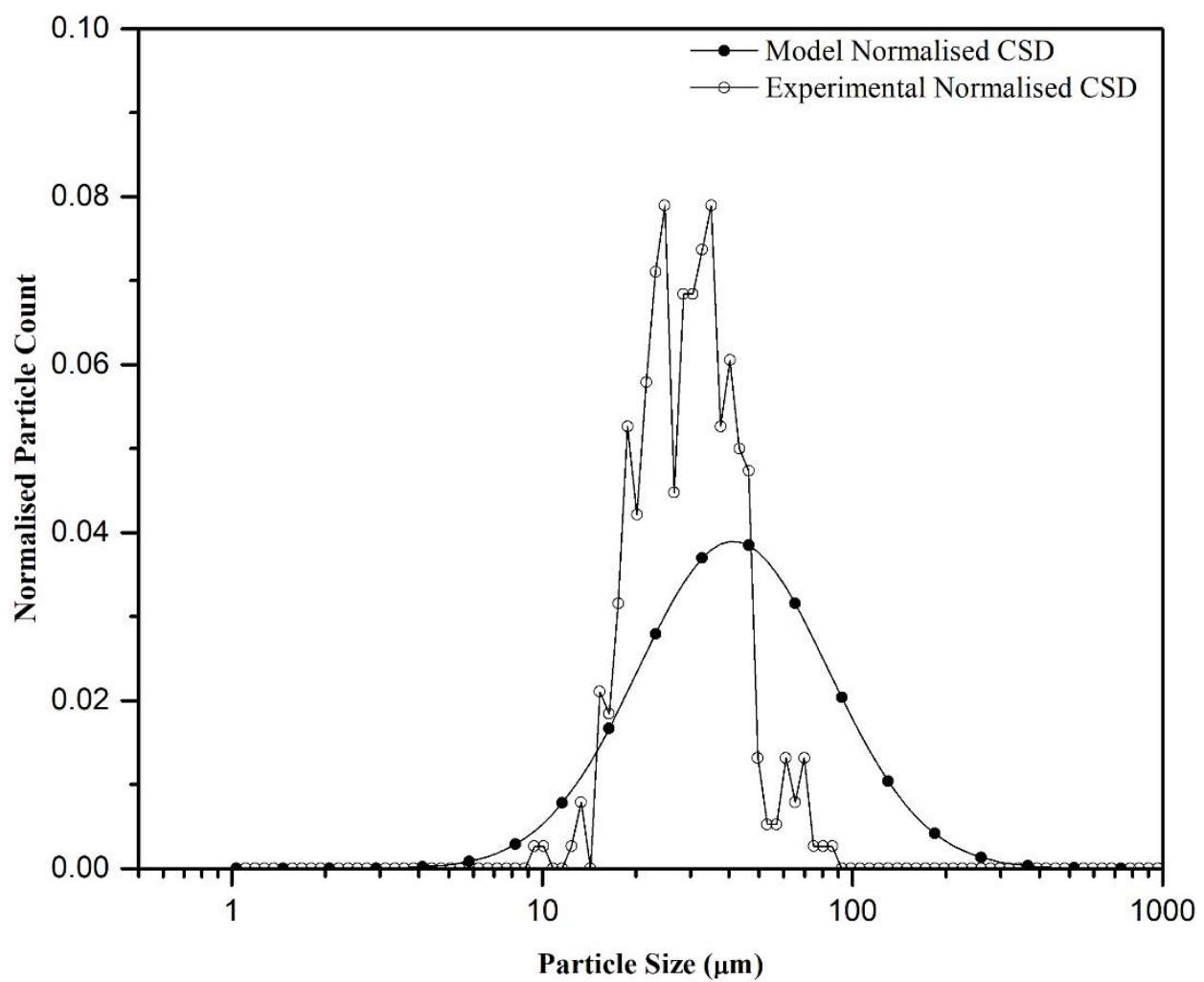


Figure 9

9(A)

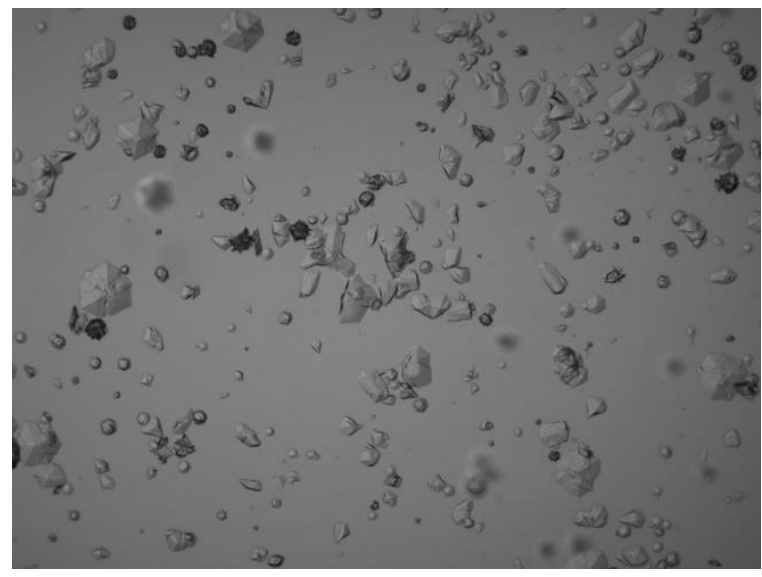

9(B)

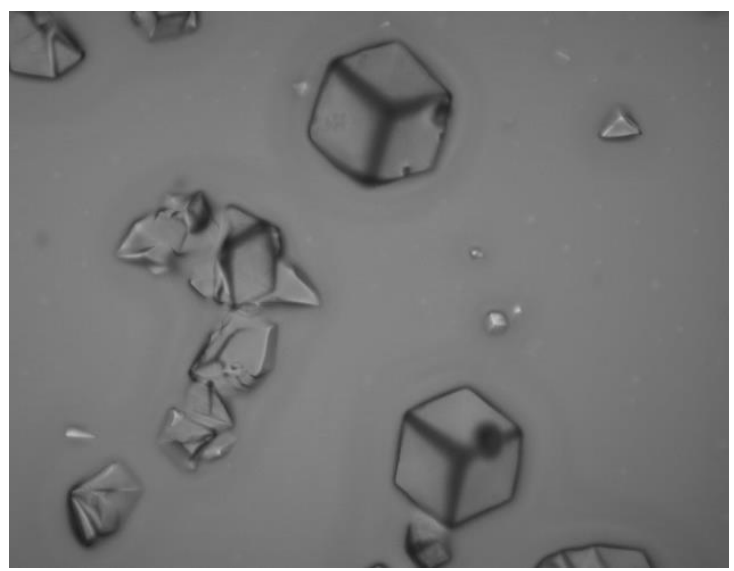

9(C)

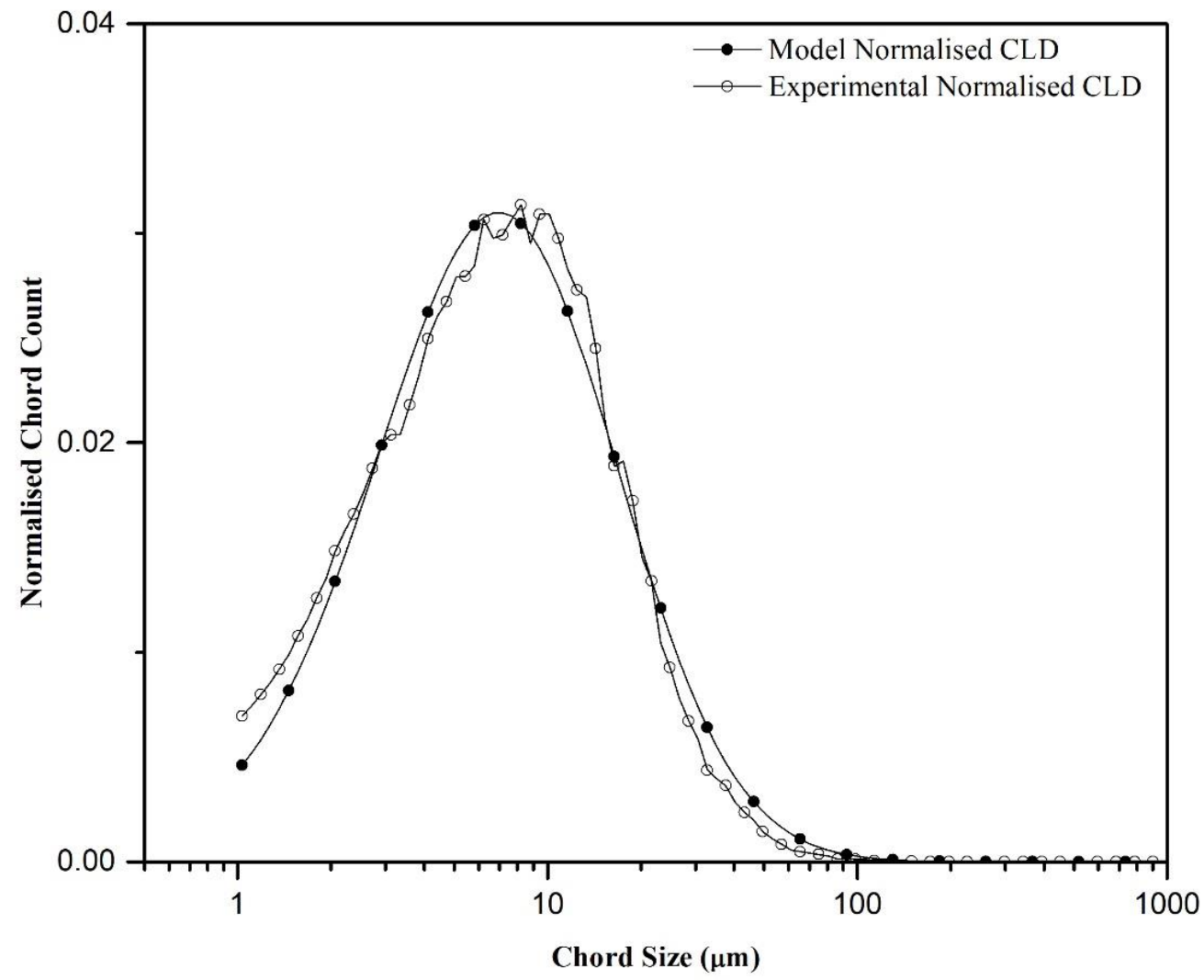


9(D)

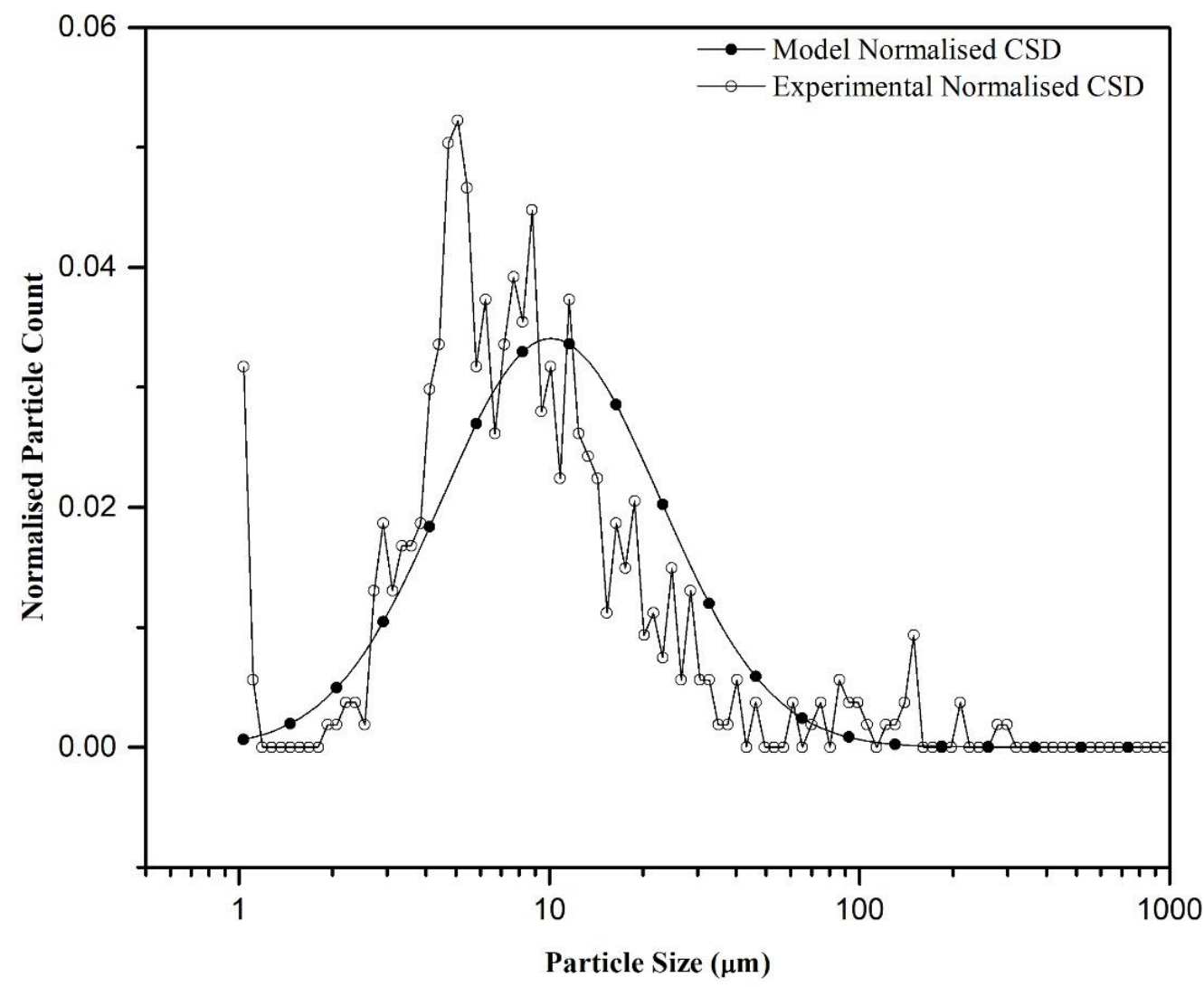


Figure 10

10(A)

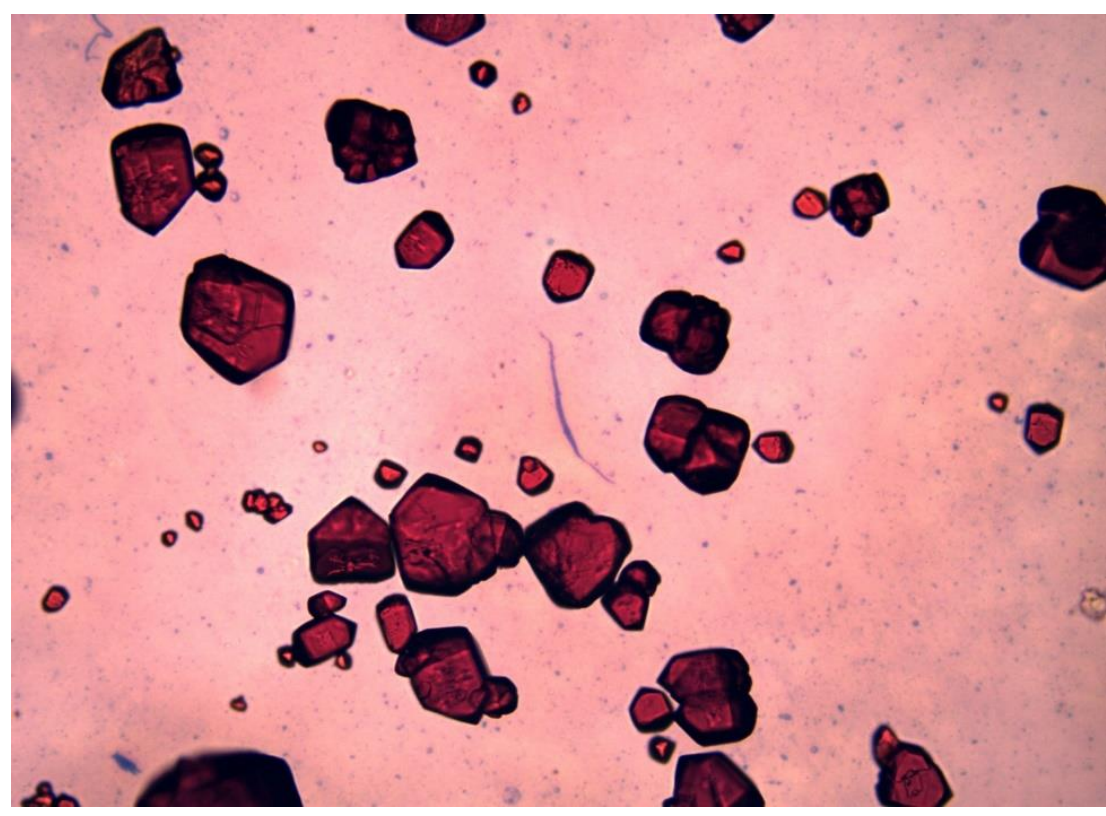

10(B)

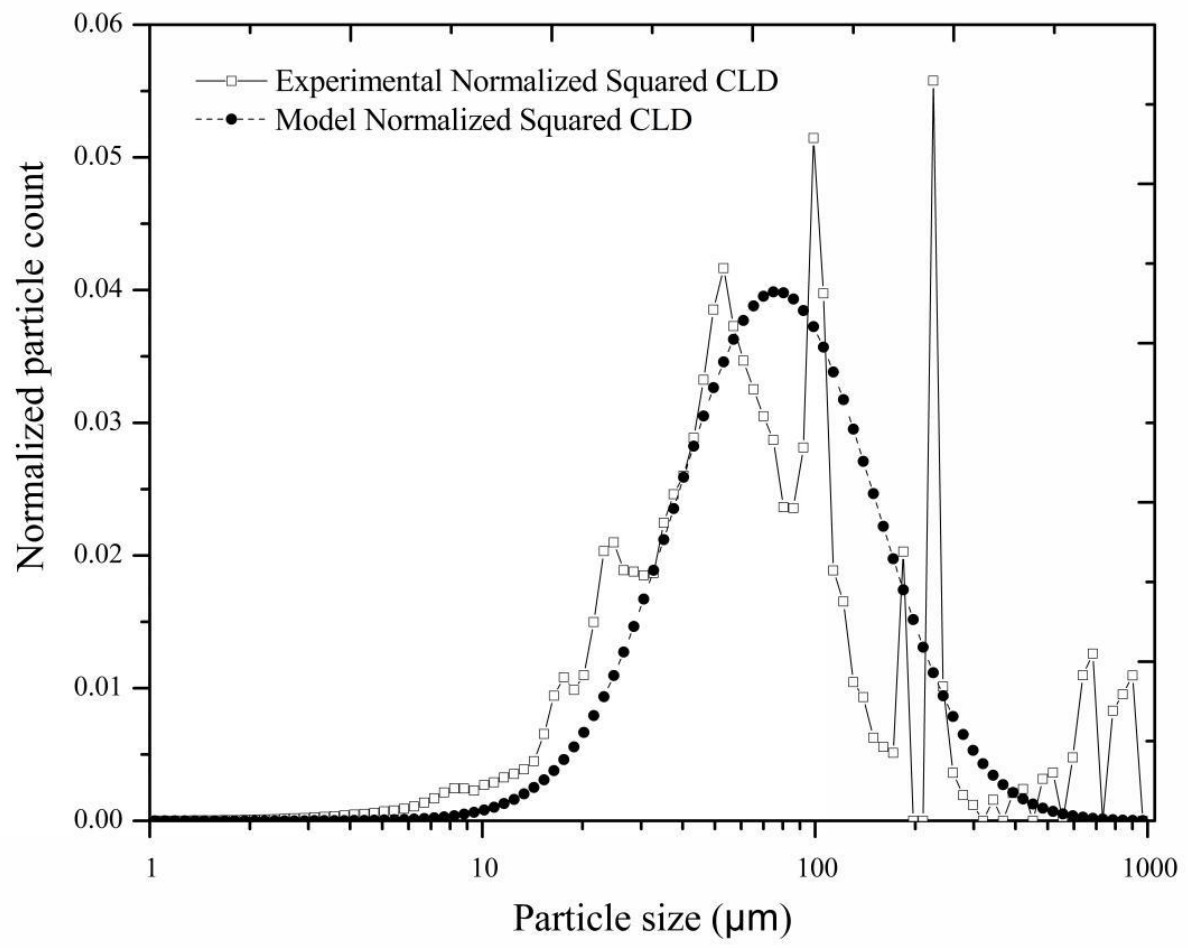


10(C)

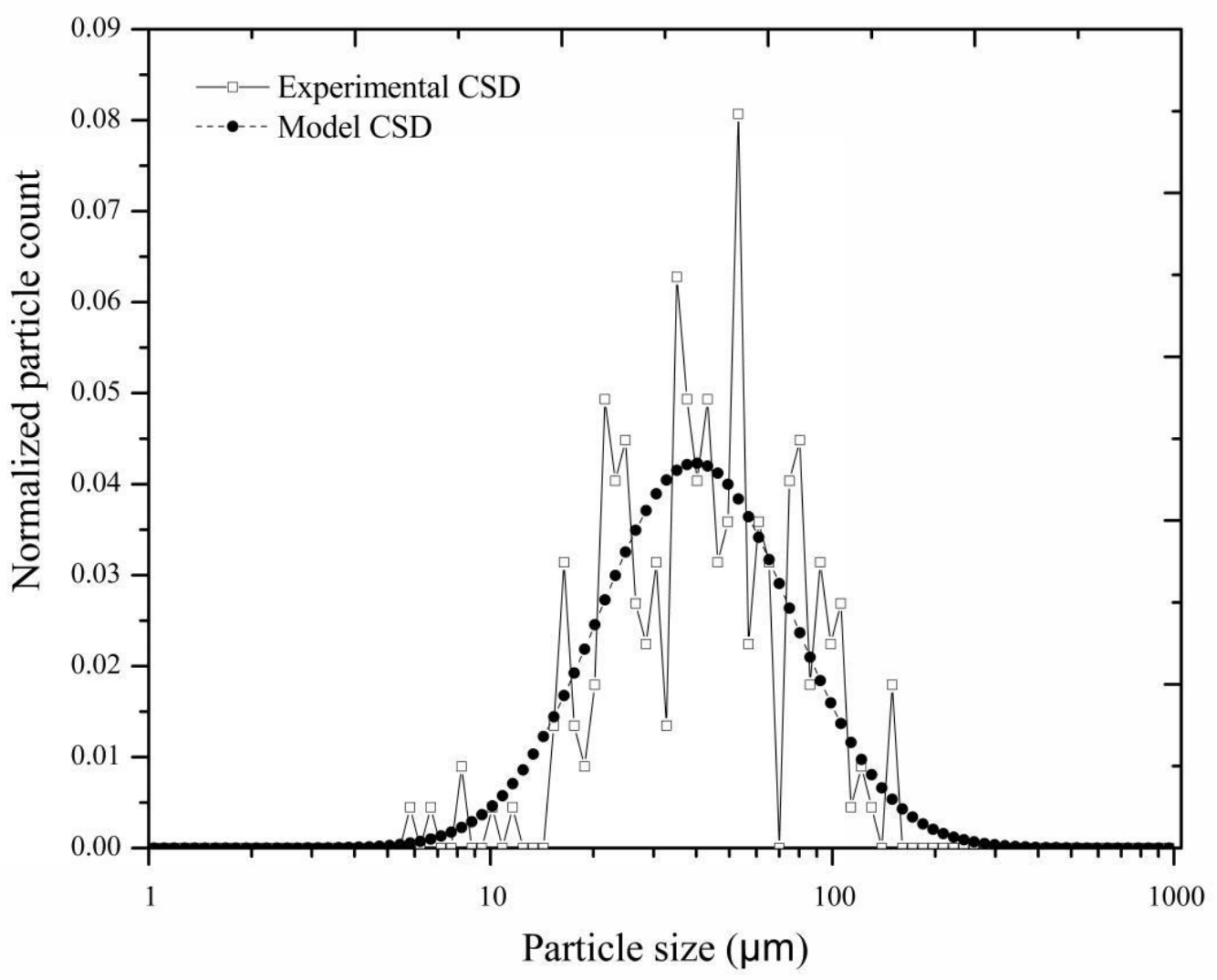


Tables

Table 1

\begin{tabular}{|c|c|c|c|c|}
\hline System & $\begin{array}{c}\text { Lysozyme } \\
\text { Conc }(\mathbf{m g} / \mathbf{m L})\end{array}$ & $\begin{array}{c}\text { Salt Conc } \\
(\mathbf{w} / \mathbf{v}) \%\end{array}$ & $\begin{array}{c}\text { Stirring } \\
\text { Speed } \\
(\mathbf{r p m})\end{array}$ & $\begin{array}{c}\text { Max. Local Energy } \\
\text { Dissipation } \\
\text { (W/kg) }\end{array}$ \\
\hline $\begin{array}{c}\text { A. Static - } \\
\text { Tetragonal Crystals }\end{array}$ & 30.00 & 5.00 & 0 & 0 \\
\hline $\begin{array}{c}\text { B. Dynamic- } \\
\text { Tetragonal Crystals }\end{array}$ & 40.00 & 3.00 & 50.00 & 0.81 \\
\hline $\begin{array}{c}\text { C. Dynamic - } \\
\text { Needle shaped } \\
\text { Crystals }\end{array}$ & 30.00 & 5.00 & 125.00 & 2.03 \\
\hline
\end{tabular}

Table 2

\begin{tabular}{|c|c|c|}
\hline Experiment & $\begin{array}{c}\text { Experimental } \\
\text { Mean crystal size } \\
(\mu \mathrm{m})\end{array}$ & $\begin{array}{c}\text { Model } \\
\text { Mean crystal size }(\mu \mathrm{m})\end{array}$ \\
\hline $\begin{array}{c}\text { Lysozyme static crystallization forming } \\
\text { tetragonal crystals }\end{array}$ & $15.67 \pm 4.67$ & $\begin{array}{c}14.10 \pm 13.56 \\
\text { SED based model }\end{array}$ \\
\hline $\begin{array}{c}\text { Lysozyme dynamic crystallization forming } \\
\text { tetragonal crystals }\end{array}$ & $18.90 \pm 5.17$ & $\begin{array}{c}33.87 \pm 57.37 \\
\text { SED based model }\end{array}$ \\
\hline $\begin{array}{l}\text { Lysozyme dynamic crystallization forming } \\
\text { needle-shaped crystals (Width measurement) }\end{array}$ & $3.26 \pm 0.49$ & $\begin{array}{c}4.95 \pm 3.84 \\
\text { Width-based model }\end{array}$ \\
\hline $\begin{array}{l}\text { Lysozyme dynamic crystallization forming } \\
\text { crystals needle-shaped crystals (Length } \\
\text { measurement) }\end{array}$ & $31.35 \pm 12.06$ & $\begin{array}{c}52.96 \pm 42.61 \\
\text { Length-based model }\end{array}$ \\
\hline Rhombohedral Insulin crystals & $15.26 \pm 29.94$ & $\begin{array}{c}14.10 \pm 13.56 \\
\text { SED based model }\end{array}$ \\
\hline Poly hydral Vitamin B12 crystals & $30.00 \pm 27.94$ & $\begin{array}{c}49.00 \pm 30.00 \\
\text { SED based model }\end{array}$ \\
\hline
\end{tabular}

